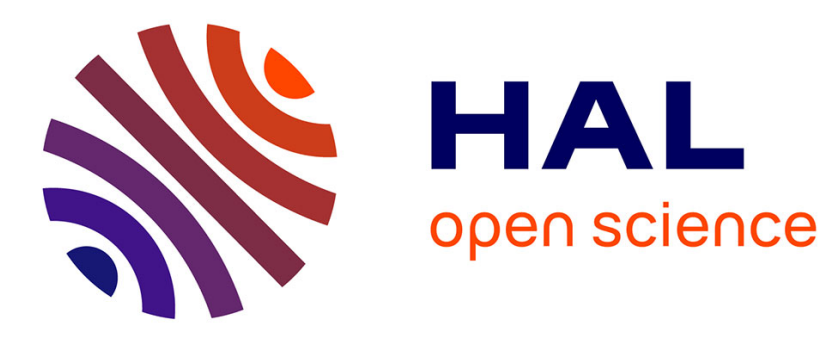

\title{
Spectral assignment for neutral-type systems and moment problems.
}

\author{
Katerina V. Sklyar, Rabah Rabah, Grigory M. Sklyar
}

\section{To cite this version:}

Katerina V. Sklyar, Rabah Rabah, Grigory M. Sklyar. Spectral assignment for neutral-type systems and moment problems.. SIAM Journal on Control and Optimization, 2015, 53 (2), pp.845-873. 10.1137/130930340. hal-01102396

\section{HAL Id: hal-01102396 \\ https://hal.science/hal-01102396}

Submitted on 12 Jan 2015

HAL is a multi-disciplinary open access archive for the deposit and dissemination of scientific research documents, whether they are published or not. The documents may come from teaching and research institutions in France or abroad, or from public or private research centers.
L'archive ouverte pluridisciplinaire HAL, est destinée au dépôt et à la diffusion de documents scientifiques de niveau recherche, publiés ou non, émanant des établissements d'enseignement et de recherche français ou étrangers, des laboratoires publics ou privés.

\section{(이) $\$$}

Distributed under a Creative Commons Attribution - NonCommercial - NoDerivatives| 4.0 


\title{
SPECTRAL ASSIGNMENT FOR NEUTRAL-TYPE SYSTEMS AND MOMENT PROBLEMS*
}

\author{
K. V. SKLYAR ${ }^{\dagger \ddagger}, R$. RABAH**,AND G. M. SKLYAR ${ }^{\dagger \ddagger}$
}

\begin{abstract}
For a large class of linear neutral-type systems the problem of assigning eigenvalues and eigenvectors is investigated, i.e. finding the system that has the given spectrum and, in some sense, allmost all eigenvectors. The solution of this problem enables vector moment problems to be considered using the construction of a neutral-type system. The exact controllability property of the system obtained gives the solution of the vector moment problem.
\end{abstract}

Key words. Neutral-type systems, eigenvalue and eigenvector assignment, moment problems.

AMS subject classifications. 93C23, 93B60, 44A60

1. Introduction. One of the central problems in control theory is that of spectral assignment. This question has been well investigated for linear finite dimensional systems, see for example [27]. It is important to emphasize that the assignment of eigenvalues is not sufficient in several cases. Sometimes, the assignment of eigenvectors or of the geometric eigenstructure is needed (see for example [11, 23, 26]). This is possible by state or output feedback using the possibility of multivariable control.

For infinite dimensional systems (delay systems, partial derivative equations) the problem is much more complicated. However, for some particular classes of infinite dimensional systems, it is possible to assign a part or whole spectrum, see for example $[4,15,22,25]$. The main motivation is the stabilizability by feedback. However, the assignment is always related to some controllability conditions. For example, the main result in [25] for the spectrum shifting is based on the exact controllability condition.

Our purpose is to investigate this kind of problem for a large class of linear delay systems of neutral-type, which may be considered in an infinite dimensional framework.

The general properties of systems of neutral-type have been widely investigated, see for example the classic works $[2,6,9]$ and references therein.

Our subject of study is the system given by the equation

$$
\dot{z}(t)=A_{-1} \dot{z}(t-1)+\int_{-1}^{0} A_{2}(\theta) \dot{z}(t+\theta) \mathrm{d} \theta+\int_{-1}^{0} A_{3}(\theta) z(t+\theta) \mathrm{d} \theta,
$$

where $z(t) \in \mathbb{R}^{n}$ and $A_{-1}, A_{2}, A_{3}$ are $n \times n$ matrices. The elements of matrices $A_{2}(\cdot)$ and $A_{3}(\cdot)$ take values in $L_{2}(-1,0)$. The neutral-type term $A_{-1} \dot{z}(t-1)$ consists of a simple delay, while the others include multiple discrete and distributed delays.

As shown in $[3,18,19]$, this system can be rewritten in the operator form

$$
\dot{x}(t)=\mathcal{A} x(t), \quad x(t)=\left(\begin{array}{c}
y(t) \\
z_{t}(\cdot)
\end{array}\right)
$$

\footnotetext{
* Some results developed here were announced in a short note published by Comptes Rendus Mathématique [24].

†Institute of Mathematics, University of Szczecin, Wielkopolska 15, 70-451 Szczecin, Poland

**IRCCyN/École des Mines de Nantes, 4 rue Alfred Kastler, BP 20722, 44307 Nantes, France

${ }^{\ddagger}$ Supported by the Polish National Science Center, grant No N514 238438.
} 
where $z_{t}(\theta)=z(t+\theta)$ and the operator $\mathcal{A}: D(\mathcal{A}) \rightarrow M_{2}=\mathbb{C}^{n} \times L_{2}\left([-1,0], \mathbb{C}^{n}\right)$, with the domain

$$
D(\mathcal{A})=\left\{\left(\begin{array}{c}
y \\
\varphi(\cdot)
\end{array}\right) \mid \varphi(\cdot) \in H^{1}\left([-1,0], \mathbb{C}^{n}\right), y=\varphi(0)-A_{-1} \varphi(-1)\right\} \subset M_{2},
$$

is given by the formula

$$
\mathcal{A}\left(\begin{array}{c}
y \\
\varphi(\cdot)
\end{array}\right)=\left(\begin{array}{c}
\int_{-1}^{0} A_{2}(\theta) \dot{\varphi}(\theta) \mathrm{d} \theta+\int_{-1}^{0} A_{3}(\theta) \varphi(\theta) \mathrm{d} \theta \\
\frac{\mathrm{d} \varphi}{\mathrm{d} \theta}(\cdot)
\end{array}\right),
$$

and is the infinitesimal generator of a $C_{0}$-semigroup [3]. This operator is noted $\widetilde{\mathcal{A}}$ instead of $\mathcal{A}$ if $A_{2}(\theta)=A_{3}(\theta) \equiv 0$. The operator $\widetilde{\mathcal{A}}$ is defined in the same domain $D(\mathcal{A})$.

It is well known that the spectral properties of this system are described by the characteristic matrix $\Delta(\lambda)$ given by

$$
\Delta(\lambda)=\lambda I-\lambda \mathrm{e}^{-\lambda} A_{-1}-\int_{-1}^{0} \lambda \mathrm{e}^{\lambda \theta} A_{2}(\theta) \mathrm{d} \theta-\int_{-1}^{0} \mathrm{e}^{\lambda \theta} A_{3}(\theta) \mathrm{d} \theta .
$$

The eigenvalues are roots of the equation $\operatorname{det} \Delta(\lambda)=0$. The eigenvectors of the system (more precisely of the functional operator model (1.2) of the system) are expressed through the kernels of the matrix $\Delta\left(\lambda_{i}\right)$, where $\lambda_{i}$ are eigenvalues.

It is noteworthy that the eigenvalues and the eigenvectors of the operator $\mathcal{A}$ are quadratically close to those of the operator $\widetilde{\mathcal{A}}$ and the latter ones are expressed through the eigenvalues and eigenvectors of the matrix $A_{-1}$. In this sense, we can say that the behavior of such systems can be described mainly by the algebraic and geometric properties of the spectrum of the matrix $A_{-1}$, (cf. $\left.[18,19]\right)$.

In parallel with the system (1.1), we consider the controlled system

$$
\dot{z}(t)=A_{-1} \dot{z}(t-1)+\int_{-1}^{0} A_{2}(\theta) \dot{z}(t+\theta) \mathrm{d} \theta+\int_{-1}^{0} A_{3}(\theta) z(t+\theta) \mathrm{d} \theta+B u,
$$

or the corresponding operator model

$$
\dot{x}(t)=\mathcal{A} x(t)+\mathcal{B} u(t),
$$

where $\mathcal{B} u=(B u, 0)^{T}$. For this controlled system, the problem of spectrum assignment has been investigated mainly in order to stabilize it by state feedback $u(t)=\mathcal{F} x(t)$, where $\mathcal{F}$ is a linear operator defined in $M_{2}$. Depending on the assumption about the operator $\mathcal{F}$ (bounded, $\mathcal{A}$-bounded, with domain $D(\mathcal{A})$, or not), and depending on the type of stabilizability needed (strong, exponential, asymptotic, regular), many important results have been obtained by several authors (see $[14,12,7,17]$ and the discussion in [20]).

In particular, when $B=I$, the identity in $\mathbb{C}^{n}$, and $A_{-1}$ has a simple spectrum $\left\{\mu_{1}, \ldots, \mu_{n}\right\}$, from the results in $[20,21,17]$, it is possible to assign arbitrarily the spectrum of $\mathcal{A}$ in the neighborhood of $\ln \mu_{m}$, where $\ln$ is the complex logarithm multifunction, by a regular feedback (the neutral term $A_{-1}$ is not modified). This consists, in fact, of the construction of matrices $A_{2}(\cdot), A_{3}(\cdot)$ to obtain the spectrum needed.

In this paper, we investigate an inverse problem: 
What conditions must a sequence of complex numbers $\{\lambda\}$ and a sequence of vectors $\{v\}$ satisfy in order to be a sequence of roots of the characteristic equation $\operatorname{det} \Delta(\lambda)=0$ and a sequence of degenerating vectors of the characteristic matrix $\Delta(\lambda)$ of equation (1.1), respectively, for some choice of matrices $A_{-1}, A_{2}(\theta), A_{3}(\theta)$ ?

The first part of the paper is devoted to this problem and is organized as follows.

We show that the assignment of eigenvectors and eigenvalues is equivalent to the existence of a linear operator $\mathcal{P}_{0}$, bounded from $D(\mathcal{A})$ (with the graph norm) to $\mathbb{C}^{n}$, defined by

$$
\mathcal{P}_{0}\left(\begin{array}{c}
y \\
\varphi(\cdot)
\end{array}\right)=\int_{-1}^{0} A_{2}(\theta) \dot{\varphi}(\theta) \mathrm{d} \theta+\int_{-1}^{0} A_{3}(\theta) \varphi(\theta) \mathrm{d} \theta
$$

such that $\mathcal{A}=\widetilde{\mathcal{A}}+\mathcal{B}_{0} \mathcal{P}_{0}$, where $\mathcal{B}_{0}=(I, 0)$, has the spectral properties needed (eigenvalues and eigenvectors).

Then, we consider the matrix $F(\lambda)=I+\mathcal{P}_{0} R(\widetilde{\mathcal{A}}, \lambda) \mathcal{B}_{0}$. We show that the eigenvalues $\lambda_{0}$ of $\mathcal{A}$ are the zeros of $\operatorname{det} F(\lambda)$ and the kernel of $\Delta\left(\lambda_{0}\right)$ is the kernel of $F\left(\lambda_{0}\right)$.

After that, using the Riesz basis property of eigenvectors of $\mathcal{A}$, we give a detailed analysis and solution of the inverse spectral problem. The main result may be summarized by the following theorem.

THEOREM 1.1. Let $\mu_{1}, \ldots, \mu_{n}$ be distinct non-zero complex scalars and $z_{1}, \ldots, z_{n}$ a linear independent set in $\mathbb{C}^{n}$. For

$$
\widetilde{\lambda}_{k}^{m}=\ln \left|\mu_{m}\right|+\mathrm{i}\left(\operatorname{Arg} \mu_{m}+2 \pi k\right), \quad m=1, \ldots, n, \quad k \in \mathbb{Z},
$$

we consider an arbitrary set of distinct complex numbers $\left\{\lambda_{k}^{m}\right\} \cup\left\{\lambda_{j}^{0}\right\}_{j=1, \ldots, n}$ such that $\sum_{k}\left|\lambda_{k}^{m}-\widetilde{\lambda}_{k}^{m}\right|^{2}<\infty, m=1, \ldots, n$ and an arbitrary set of vectors $\left\{w_{k}^{m}\right\} \cup\left\{w_{j}^{0}\right\}$ satisfying $\sum_{k}\left\|w_{k}^{m}-z_{m}\right\|^{2}<\infty, m=1, \ldots, n$. Then, there are matrices $A_{-1}, A_{2}(\theta), A_{3}(\theta)$ such that:

i) The scalars $\left\{\lambda_{k}^{m}, \lambda_{j}^{0}\right\}$ are simple roots of the equation $\operatorname{det} \Delta(\lambda)=0$;

ii) $w_{k}^{m^{*}} \Delta\left(\lambda_{k}^{m}\right)=0, m=1, \ldots, n ; k \in \mathbb{Z}$ and $w_{j}^{0^{*}} \Delta\left(\lambda_{j}^{0}\right)=0, j=1 \ldots, n$; except perhaps for a finite number of vectors $w_{k}^{m}$. For this last case, there are vectors $\widehat{w}_{k}^{m}$ arbitrarily close to the vectors $w_{m}: \widehat{w}_{k}^{m^{*}} \Delta\left(\lambda_{k}^{m}\right)=0$.

This theorem was announced in Comptes Rendus Mathematique [24].

As an application of this spectral assignment problem, we consider a vector moment problem via the solution of the exact controllability property for a corresponding neutral-type system (1.4) by an approach developed in [16]. This is the main purpose of the second part of the paper, namely Section 6 .

A classic trigonometric moment problem is a set of equations

$$
\int_{0}^{T} \mathrm{e}^{\gamma_{i} t} v(t) \mathrm{d} t=c_{i}, \quad i \in \mathbb{N},
$$

where $\gamma_{i} \in \mathbb{C}$, the sequence $\left\{c_{i}\right\}$ is given and the function $v \in L_{2}(0, T)$ is unknown. The goal is to give conditions of solvability for all $\left\{c_{i}\right\} \in \ell_{2}$ and find a minimal interval of this solvability. Several works are devoted to this important problem. The approach is often based on the study of exponential families, which dates back to the works of Paley and Wiener [13]. Several authors have obtained important results in 
this area (see for example $[10,8,28]$ ). In [1], there are several practical frameworks and useful results for our investigation.

Two of the authors of the present paper showed in [16] that the exact nullcontrollability problem of the controlled neutral-type system leads to a vector moment problem. This problem may be formulated in some particular cases as

$$
s_{k}^{m}=\int_{0}^{T} \mathrm{e}^{\lambda_{k}^{m} t}\left(b_{k, m}^{1} u_{1}(t)+\ldots+b_{k, m}^{r} u_{r}(t)\right) \mathrm{d} t, \quad k \in \mathbb{Z}, m=1, \ldots, n .
$$

What is important in this context is the fact that the numbers $\lambda_{k}^{m}$ are the roots of the equation $\operatorname{det} \Delta(\lambda)=0$ and the coefficients $b_{k, m}^{j}$ are expressed via the vectors $w_{k}^{m}, w_{k}^{m *} \Delta\left(\lambda_{k}^{m}\right)=0$ by the formula $b_{k, m}^{j}=\left\langle b_{j}, w_{k}^{m}\right\rangle$, where $B=\left(b_{1}, \ldots, b_{r}\right)$. Thus, the problem formulated above of spectral (eigenvalues-eigenvectors) assignment defines completely the vector moment corresponding to the problem of exact null-controllability. Roughly speaking, the null-controllability problem can be considered a problem of eigenfrequency extinguishing, where the sequence $\lambda_{k}^{m}$ means the chosen frequencies and coefficients $b_{k, m}^{j}$ (vectors $w_{k}^{m}$ ) determine the contribution of the particular control inputs to the extinguishing of the frequency $\lambda_{k}^{m}$.

For those moment problems (1.5) that are related to the exact controllability property of the controlled neutral-type system, the solvability conditions and the minimal interval of solvability (minimal time of exact controllability) are found.

Here we consider a more general problem:

Starting with an abstract moment problem (1.5), and using the result of the first part, give conditions of the existence of a neutral-type system for which the exact controllability condition is equivalent (almost equivalent in some sense) to the vector moment problem.

This enables the solvability condition and the minimal interval to be given in the general case.

An illustrative example is provided.

2. The state operator as a perturbation. The state operator $\mathcal{A}$ can be considered a perturbation of the operator $\widetilde{\mathcal{A}}$, namely

$$
\mathcal{A}\left(\begin{array}{c}
y \\
\varphi(\cdot)
\end{array}\right)=\widetilde{\mathcal{A}}\left(\begin{array}{c}
y \\
\varphi(\cdot)
\end{array}\right)+\left(\begin{array}{c}
\int_{-1}^{0} A_{2}(\theta) \dot{\varphi}(\theta) \mathrm{d} \theta+\int_{-1}^{0} A_{3}(\theta) \varphi(\theta) \mathrm{d} \theta \\
0
\end{array}\right) .
$$

Let $\mathcal{B}_{0}: \mathbb{C}^{n} \rightarrow M_{2}$ be given by

$$
\mathcal{B}_{0} y=\left(\begin{array}{l}
y \\
0
\end{array}\right)
$$

and $\mathcal{P}_{0}: D(\mathcal{A}) \rightarrow \mathbb{C}^{n}$ by

$$
\mathcal{P}_{0}\left(\begin{array}{c}
y \\
\varphi(\cdot)
\end{array}\right)=\int_{-1}^{0} A_{2}(\theta) \dot{\varphi}(\theta) \mathrm{d} \theta+\int_{-1}^{0} A_{3}(\theta) \varphi(\theta) \mathrm{d} \theta .
$$

Then $\mathcal{A}=\widetilde{\mathcal{A}}+\mathcal{B}_{0} \mathcal{P}_{0}$. Denote by $X_{\mathcal{A}}$ the set $D(\mathcal{A})$ endowed with the graph norm.

LEMma 2.1. The linear bounded operator $\mathcal{P}_{0}$ browses the set of all linear bounded operators $\mathcal{L}\left(X_{\mathcal{A}}, \mathbb{C}^{n}\right)$ as $A_{2}(\cdot), A_{3}(\cdot)$ run over the set of $n \times n$ matrices with components from $L_{2}[-1,0]$. 
Proof. An arbitrary linear operator $Q$ from $\mathcal{L}\left(X_{\mathcal{A}}, \mathbb{C}^{n}\right)$ can be presented as

$$
\begin{aligned}
Q\left(\begin{array}{c}
y \\
\varphi(\cdot)
\end{array}\right) & =Q_{1} y+Q_{2} \varphi(\cdot) \\
& =Q_{1}\left(\varphi(0)-A_{-1} \varphi(-1)\right)+\int_{-1}^{0} \widehat{A}_{2}(\theta) \dot{\varphi}(\theta) \mathrm{d} \theta+\int_{-1}^{0} \widehat{A}_{3}(\theta) \varphi(\theta) \mathrm{d} \theta
\end{aligned}
$$

where $\widehat{A}_{2}(\cdot), \widehat{A}_{3}(\cdot)$ are $(n \times n)$-matrices with component from $L_{2}[-1,0]$ and $Q_{1}$ is a $(n \times n)$ matrix. Let us observe that

$$
\begin{aligned}
\varphi(-1) & =\int_{-1}^{0} \theta \dot{\varphi}(\theta) \mathrm{d} \theta+\int_{-1}^{0} \varphi(\theta) \mathrm{d} \theta \\
\varphi(0) & =\int_{-1}^{0}(\theta+1) \dot{\varphi}(\theta) \mathrm{d} \theta+\int_{-1}^{0} \varphi(\theta) \mathrm{d} \theta
\end{aligned}
$$

and denote

$$
\begin{aligned}
& A_{2}(\theta)=\widehat{A}_{2}(\theta)+(\theta+1) Q_{1}-\theta Q_{1} A_{-1}, \\
& A_{3}(\theta)=\widehat{A}_{3}(\theta)+Q_{1}-Q_{1} A_{-1} .
\end{aligned}
$$

Then, with these notations, the operator $Q$ may be written as

$$
Q\left(\begin{array}{c}
y \\
\varphi(\cdot)
\end{array}\right)=\int_{-1}^{0} A_{2}(\theta) \dot{\varphi}(\theta) \mathrm{d} \theta+\int_{-1}^{0} A_{3}(\theta) \varphi(\theta) \mathrm{d} \theta .
$$

Hence formula (2.1) describes all the operators from $\mathcal{L}\left(X_{\mathcal{A}}, \mathbb{C}^{n}\right)$.

3. An equation for eigenvalues and eigenvectors: the spectral equation. Consider the operator $\mathcal{A}=\widetilde{\mathcal{A}}+\mathcal{B}_{0} \mathcal{P}_{0}$ and assume that $\lambda_{0}$ is an eigenvalue of $\mathcal{A}$ and $x_{0}$ is a corresponding eigenvector, i.e.

$$
\left(\widetilde{\mathcal{A}}+\mathcal{B}_{0} \mathcal{P}_{0}\right) x_{0}=\lambda_{0} x_{0} .
$$

Let us assume further that $\lambda_{0}$ does not belong to the spectrum of $\widetilde{\mathcal{A}}$ and denote by $R\left(\widetilde{\mathcal{A}}, \lambda_{0}\right)=\left(\widetilde{\mathcal{A}}-\lambda_{0} I\right)^{-1}$. With this notation, (3.1) reads as

$$
x_{0}+R\left(\widetilde{\mathcal{A}}, \lambda_{0}\right) \mathcal{B}_{0} \mathcal{P}_{0} x_{0}=0 .
$$

Let us notice that $v_{0}=\mathcal{P}_{0} x_{0} \neq 0$, because $\lambda_{0} \notin \sigma(\widetilde{\mathcal{A}})$. Then applying operator $\mathcal{P}_{0}$ to the left-hand side of $(3.2)$ we obtain

$$
v_{0}+\mathcal{P}_{0} R\left(\widetilde{\mathcal{A}}, \lambda_{0}\right) \mathcal{B}_{0} v_{0}=0
$$

This equality means that $\lambda_{0}$ is a point of singularity of the matrix-valued function

$$
F(\lambda)=I+\mathcal{P}_{0} R(\widetilde{\mathcal{A}}, \lambda) \mathcal{B}_{0}
$$

and $v_{0}$ is a vector degenerating $F\left(\lambda_{0}\right)$ from the right.

In what follows, the left kernel of the matrix $F\left(\lambda_{0}\right)$ plays a significant role. Let us give a characterization of the vectors of this subspace.

Proposition 3.1. Let $\lambda_{0}$ not belong to $\sigma(\widetilde{\mathcal{A}})$. Then, the pair $\left(\lambda_{0}, w_{0}\right), w_{0} \in$ $\mathbb{C}^{n}, w_{0} \neq 0$, satisfies the spectral equation

$$
w_{0}^{*} F\left(\lambda_{0}\right)=0 .
$$


if and only if $\lambda_{0}$ is a root of the characteristic equation det $\Delta(\lambda)=0$, where $\Delta(\lambda)$ is given in (1.3) and $w_{0}{ }^{*}$ is a row-vector in the left kernel of $\Delta\left(\lambda_{0}\right): w_{0}{ }^{*} \Delta\left(\lambda_{0}\right)=0$.

Proof. In order to describe the vector $w_{0}$, let us first find another form for the matrix $F(\lambda)$. For any $v \in \mathbb{C}^{n}$ we denote

$$
R(\widetilde{\mathcal{A}}, \lambda) \mathcal{B}_{0} v=\left(\begin{array}{c}
y \\
\varphi(\cdot)
\end{array}\right), \quad \lambda \notin \sigma(\widetilde{\mathcal{A}})
$$

then

$$
(\widetilde{\mathcal{A}}-\lambda I)\left(\begin{array}{c}
y \\
\varphi(\cdot)
\end{array}\right)=\mathcal{B}_{0} v=\left(\begin{array}{l}
v \\
0
\end{array}\right)
$$

This gives $v=-\lambda y$ and $\frac{\mathrm{d} \varphi(\theta)}{\mathrm{d} \theta}-\lambda \varphi(\theta)=0$. As $\left(\begin{array}{c}y \\ \varphi(\cdot)\end{array}\right) \in D(\widetilde{\mathcal{A}})$, we obtain $y=$ $\varphi(0)-A_{-1} \varphi(-1)$. Therefore

$$
\varphi(\theta)=d \mathrm{e}^{\lambda \theta}, \quad d \in \mathbb{C}^{n}
$$

and

$$
v=-\lambda\left(I-\mathrm{e}^{-\lambda} A_{-1}\right) d
$$

Since the matrix $\left(I-\mathrm{e}^{-\lambda} A_{-1}\right)$ is invertible $(\lambda \notin \sigma(\widetilde{\mathcal{A}}))$, we obtain

$$
\varphi(\theta)=-\frac{\mathrm{e}^{\lambda \theta}}{\lambda}\left(I-\mathrm{e}^{-\lambda} A_{-1}\right)^{-1} v
$$

and hence

$$
R(\widetilde{\mathcal{A}}, \lambda) \mathcal{B}_{0} v=\left(\begin{array}{c}
y \\
-\frac{\mathrm{e}^{\lambda \theta}}{\lambda}\left(I-\mathrm{e}^{-\lambda} A_{-1}\right)^{-1} v
\end{array}\right)
$$

This formula and (2.1) implies

$$
v+\mathcal{P}_{0} R(\widetilde{\mathcal{A}}, \lambda) \mathcal{B}_{0} v=v-\left(\int_{-1}^{0} \mathrm{e}^{\lambda \theta} A_{2}(\theta) \mathrm{d} \theta-\int_{-1}^{0} \frac{\mathrm{e}^{\lambda \theta}}{\lambda} A_{3}(\theta) \mathrm{d} \theta\right)\left(I-\mathrm{e}^{-\lambda} A_{-1}\right)^{-1} v
$$

and hence

$$
F(\lambda) v=\Delta(\lambda) \frac{\left(I-\mathrm{e}^{-\lambda} A_{-1}\right)^{-1}}{\lambda} v
$$

Thus, the equality (3.3) for $\lambda_{0} \notin \sigma(\widetilde{\mathcal{A}})$ is equivalent to $w_{0}{ }^{*} \Delta\left(\lambda_{0}\right)=0$.

Proposition 3.1 means that the equation $w^{*} F(\lambda)=0$ can be considered an equation whose roots $(\lambda, w)=\left(\lambda_{0}, w_{0}\right)$ describe all eigenvalues and (right) eigenvectors of the characteristic matrix $\Delta(\lambda)$. It is called the spectral equation.

4. A component-wise representation of the spectral equation. We need a more detailed expression of the spectral equation (3.3).

Let us recall the spectral properties of operators $\widetilde{\mathcal{A}}$ and $\widetilde{\mathcal{A}}^{*}$ obtained in $[18,19]$. We will assume that the matrix $A_{-1}$ has simple non-zero eigenvalues $\mu_{1}, \mu_{2}, \mu_{3}, \ldots, \mu_{n}$. In this case the spectrum $\sigma(\widetilde{\mathcal{A}})$ consists of simple eigenvalues which we denote by

$$
\tilde{\lambda}_{k}^{m}=\ln \left|\mu_{m}\right|+\mathrm{i}\left(\operatorname{Arg} \mu_{m}+2 \pi k\right), \quad m=1, \ldots, n, k \in \mathbb{Z},
$$


and of eigenvalue $\underset{\tilde{\lambda}_{0}}{\widetilde{\lambda}_{0}}=0$. First, assume $\mu_{m} \neq 1, m=1, \ldots, n$. Then, the eigenvectors corresponding to $\tilde{\lambda}_{k}^{m}$ are of the form

$$
\widetilde{\varphi}_{k}^{m}=\left(\begin{array}{c}
0 \\
\mathrm{e}^{\widetilde{\lambda}_{k}^{m} \theta} y_{m}
\end{array}\right), \quad k \in \mathbb{Z}, m=1, \ldots, n
$$

where $y_{1}, \ldots, y_{n}$ are eigenvectors of $A_{-1}$ corresponding to $\mu_{1}, \mu_{2}, \ldots, \mu_{n}$. The eigenspace corresponding to $\widetilde{\lambda}_{0}=0$ is $n$ dimensional and its basis is

$$
\widetilde{\varphi}_{j}^{0}=\left(\begin{array}{c}
\left(1-\mu_{j}\right) y_{j} \\
y_{j}
\end{array}\right), \quad j=1, \ldots, n
$$

If some $\mu_{m}$ say $\mu_{1}$ equals 1 , then $\widetilde{\lambda}_{0}^{1}=\widetilde{\lambda}_{0}=0$. In this case, the eigenspace corresponding to 0 is $(n+1)$-dimensional and its basis consists of $n$ eigenvectors $\widetilde{\varphi}_{j}^{0}, j=1, \ldots, n$, given by (4.2), and one root-vector

$$
\widetilde{\varphi}_{0}^{1}=\left(\begin{array}{c}
y_{1} \\
\theta y_{1}
\end{array}\right), \quad \widetilde{\mathcal{A}} \widetilde{\varphi}_{0}^{1}=\widetilde{\varphi}_{1}^{0}
$$

All the vectors $\widetilde{\varphi}_{k}^{m}$ given by (4.1), except $\widetilde{\varphi}_{1}^{0}$, are still eigenvectors of $\widetilde{\mathcal{A}}$ and correspond to eigenvalues $\widetilde{\lambda}_{k}^{m}$. In both cases the family $\Phi=\left\{\widetilde{\varphi}_{k}^{m}\right\} \cup\left\{\widetilde{\varphi}_{j}^{0}\right\}$ forms a Riesz basis in the space $M_{2}$. Denote by $\Psi=\left\{\widetilde{\psi}_{k}^{m}\right\} \cup\left\{\widetilde{\psi}_{j}^{0}\right\}$ the bi-orthogonal basis to $\Phi$. Then

$$
\widetilde{\psi}_{k}^{m}=\left(\begin{array}{c}
z_{m} / \overline{\widetilde{\lambda}_{k}^{m}} \\
\mathrm{e}^{-\overline{\widetilde{\lambda}_{k}^{m}} \theta} z_{m}
\end{array}\right), \quad m=1, \ldots, n, k \in \mathbb{Z} \text { if } \widetilde{\lambda}_{0}^{1} \neq 0, \widetilde{\psi}_{0}^{1}=\left(\begin{array}{c}
z_{1} \\
0
\end{array}\right), \text { if } \widetilde{\lambda}_{0}^{1}=0,
$$

where $\overline{\widetilde{\lambda}}$ is the complex conjugate of $\widetilde{\lambda}$. Here $z_{m}$ are eigenvectors of matrix $A_{-1}^{*}$ biorthogonal to the eigenvectors $y_{m}$ of $A_{-1}$, i.e. such that $\left\langle y_{i}, z_{j}\right\rangle=\delta_{i j}$. It is easy to see that $\widetilde{\psi}_{k}^{m}$ are eigenvectors of the operator $\widetilde{\mathcal{A}}^{*}$ corresponding to eigenvalues $\overline{\widetilde{\lambda}_{k}^{m}}$.

In order to obtain a component-wise expression of the spectral equation (3.3), we need to rewrite the matrix $F\left(\lambda_{0}\right)$ in the basis $\Phi$ and the vector $w_{0}^{*}$ in the basis $\left\{z_{m}, m=1, \ldots, n\right\}$.

In what follows, we shall use the notation

$$
\widetilde{\beta}_{k}^{m}=\left\{\begin{array}{ll}
\widetilde{\lambda}_{k}^{m}, & \widetilde{\lambda}_{k}^{m} \neq 0, \\
1, & \widetilde{\lambda}_{k}^{m}=0,
\end{array} \quad m=1, \ldots, n, k \in \mathbb{Z}\right.
$$

Let us observe that the expression $w_{0}^{*} \mathcal{P}_{0}$ is a linear bounded functional on the space $X_{\mathcal{A}}$, i.e. $w_{0}^{*} \mathcal{P}_{0} \in \mathcal{L}\left(X_{\mathcal{A}}, \mathbb{C}\right)$. The representation of $w_{0}^{*}$ in the basis $z_{j}$ is as follows:

$$
w_{0}^{*}=\sum_{j} \alpha_{j} z_{j}^{*} .
$$

Consider now $n$ functionals $z_{j}^{*} \mathcal{P}_{0} \in \mathcal{L}\left(X_{\mathcal{A}}, \mathbb{C}\right), j=1, \ldots, n$. They can be decomposed in the basis $\Psi$ :

$$
z_{j}^{*} \mathcal{P}_{0}=\sum_{k, m} p_{k, m}^{j} \widetilde{\psi}_{k}^{m}+\sum_{i} \widetilde{p}_{i}^{j} \widetilde{\psi}_{i}^{0}
$$


where

$$
\sum_{k}\left|\frac{p_{k, m}^{j}}{\widetilde{\beta}_{k}^{m}}\right|^{2}<\infty, \quad m=1, \ldots, n
$$

Proposition 4.1. The pair $\left(\lambda_{0}, w_{0}\right)$ satisfies the spectral equation (3.3) if and only if the following system of $n$ equations is verified:

$$
\alpha_{m}=-\sum_{\substack{k \in \mathbb{Z} \\ j=1, \ldots, n}} \alpha_{j}\left(\frac{p_{k, m}^{j}}{\widetilde{\beta}_{k}^{m}} \times \frac{1}{\widetilde{\lambda}_{k}^{m}-\lambda_{0}}\right), \quad m=1, \ldots, n
$$

where, for any fixed couple $m, j$, the needed n-tuple $\left\{p_{k, m}^{j}\right\}$ satisfies (4.4).

Proof. Let us first rewrite the expression $R\left(\widetilde{\mathcal{A}}, \lambda_{0}\right) \mathcal{B}_{0}$. We present $\mathcal{B}_{0}$ as a matrix $\mathcal{B}_{0}=\left(\boldsymbol{b}_{1}^{0}, \boldsymbol{b}_{2}^{0}, \ldots, \boldsymbol{b}_{n}^{0}\right)$ with infinite columns $\boldsymbol{b}_{i}^{0}$ which are vectors from $M_{2}$ of the form

$$
\boldsymbol{b}_{i}^{0}=\left(\begin{array}{c}
e_{i} \\
0
\end{array}\right), \quad i=1, \ldots, n,
$$

where $e_{i}$ is the canonical basis of $\mathbb{C}^{n}$. Then

$$
\boldsymbol{b}_{i}^{0}=\sum_{k, m}\left\langle\boldsymbol{b}_{i}^{0}, \widetilde{\psi}_{k}^{m}\right\rangle \widetilde{\varphi}_{k}^{m}+\sum_{j}\left\langle\boldsymbol{b}_{i}^{0}, \widetilde{\psi}_{j}^{0}\right\rangle \widetilde{\varphi}_{j}^{0}, \quad i=1, \ldots, n,
$$

and in the case $\mu_{m} \neq 1, m=1, \ldots, n$, we have

$$
R\left(\widetilde{\mathcal{A}}, \lambda_{0}\right) \boldsymbol{b}_{i}^{0}=\sum_{k, m} \frac{1}{\widetilde{\lambda}_{k}^{m}-\lambda_{0}}\left\langle\boldsymbol{b}_{i}^{0}, \widetilde{\psi}_{k}^{m}\right\rangle \widetilde{\varphi}_{k}^{m}-\sum_{j} \frac{1}{\lambda_{0}}\left\langle\boldsymbol{b}_{i}^{0}, \widetilde{\psi}_{j}^{0}\right\rangle \widetilde{\varphi}_{j}^{0}, \quad i=1, \ldots, n .
$$

Taking into account the form of eigenvectors (4.3) we find that for all $m=1, \ldots, n$

$$
\left(\left\langle\boldsymbol{b}_{1}^{0}, \widetilde{\psi}_{k}^{m}\right\rangle, \ldots,\left\langle\boldsymbol{b}_{n}^{0}, \widetilde{\psi}_{k}^{m}\right\rangle\right)=\frac{1}{\widetilde{\lambda}_{k}^{m}}\left(\left\langle e_{1}, z_{m}\right\rangle, \ldots,\left\langle e_{n}, z_{m}\right\rangle\right)=z_{m}^{*} / \widetilde{\lambda}_{k}^{m}
$$

Thus, we obtain

(4.6) $R\left(\widetilde{\mathcal{A}}, \lambda_{0}\right) \mathcal{B}_{0}=\sum_{k, m} \frac{1}{\widetilde{\lambda}_{k}^{m}-\lambda_{0}} \times \frac{1}{\widetilde{\lambda}_{k}^{m}} \widetilde{\varphi}_{k}^{m} z_{m}^{*}-\sum_{j} \frac{1}{\lambda_{0}} \widetilde{\varphi}_{j}^{0}\left(\left\langle\boldsymbol{b}_{1}^{0}, \widetilde{\psi}_{j}^{0}\right\rangle, \ldots,\left\langle\boldsymbol{b}_{n}^{0}, \widetilde{\psi}_{j}^{0}\right\rangle\right)$

if all the numbers $\widetilde{\lambda}_{k}^{m} \neq 0$. If $\mu_{1}=1$ and $\widetilde{\lambda}_{0}^{1}=0$, then in the sum (4.6) the term corresponding to $k=1, m=1$, is replaced by

$$
\left(\frac{1}{\widetilde{\lambda}_{0}^{1}-\lambda_{0}} \widetilde{\varphi}_{0}^{1}-\frac{1}{\left(\widetilde{\lambda}_{0}^{1}-\lambda_{0}\right)^{2}} \widetilde{\varphi}_{1}^{0}\right) z_{1}^{*}
$$

In what follows, we shall assume $\widetilde{p}_{i}^{j}=0, i=1, \ldots, n$. This means that from now on we consider perturbations $\mathcal{P}_{0}$ satisfying the condition

$$
\int_{-1}^{0} A_{3}(\theta) \mathrm{d} \theta=0
$$


Then we have

$$
w_{0}^{*} \mathcal{P}_{0}=\sum_{j} \alpha_{j} z_{j}^{*} \mathcal{P}_{0}=\sum_{j} \alpha_{j} \sum_{k, m} p_{k, m}^{j} \widetilde{\psi}_{k}^{m}
$$

From this relation and expression (4.6) we obtain the equality

$$
\begin{aligned}
w_{0}^{*} \mathcal{P}_{0} \mathcal{R}\left(\widetilde{\mathcal{A}}, \lambda_{0}\right) \mathcal{B}_{0} & =\sum_{m}\left\langle\sum_{k} \frac{1}{\widetilde{\beta}_{k}^{m}} \times \frac{1}{\widetilde{\lambda}_{k}^{m}-\lambda_{0}} \widetilde{\varphi}_{k}^{m}, \sum_{j} \overline{\alpha_{j}} \sum_{k_{0}, m_{0}} \bar{\beta}_{k_{0}, m_{0}}^{j} \widetilde{\psi}_{k_{0}}^{m_{0}}\right\rangle z_{m}^{*} \\
& =\sum_{j} \alpha_{j} \sum_{k_{0}, m_{0}} \frac{p_{k, m}^{j}}{\widetilde{\beta}_{k}^{m}} \times \frac{1}{\widetilde{\lambda}_{k}^{m}-\lambda_{0}} z_{m}^{*} .
\end{aligned}
$$

With these notations, equation (3.3) reads

$$
0=w_{0}^{*}\left(I+\mathcal{P}_{0} \mathcal{R}\left(\tilde{\mathcal{A}}, \lambda_{0}\right) \mathcal{B}_{0}\right)=\sum_{m} \alpha_{m} z_{m}^{*}+\sum_{m}\left(\sum_{j, k} \alpha_{j} \frac{p_{k, m}^{j}}{\widetilde{\beta}_{k}^{m}} \times \frac{1}{\widetilde{\lambda}_{k}^{m}-\lambda_{0}}\right) z_{m}^{*}
$$

Thus, the condition for a pair $\left(\lambda_{0}, w_{0}\right)$ to satisfy the spectral equation can be rewritten in the form of the following system of $n$ equations:

$$
\alpha_{m}=-\sum_{\substack{k \in \mathbb{Z} \\ j=1, \ldots, n}} \alpha_{j}\left(\frac{p_{k, m}^{j}}{\widetilde{\beta}_{k}^{m}} \times \frac{1}{\widetilde{\lambda}_{k}^{m}-\lambda_{0}}\right), \quad m=1, \ldots, n
$$

where, for any fixed couple $m, j$, the needed $n$-tuple $\left\{p_{k, m}^{j}\right\}$ satisfies (4.4).

5. Conditions for the spectral assignment. Now we discuss the following question:

What conditions must a sequence of complex numbers $\{\lambda\}$ and a sequence of vectors $\{v\}$ satisfy in order to be a sequence of roots of the characteristic equation $\operatorname{det} \Delta(\lambda)=0$ and a sequence of degenerating vectors of the characteristic matrix $\Delta(\lambda)$ of equation (1.1), respectively, for some choice of matrices $A_{-1}, A_{2}(\theta), A_{3}(\theta)$ ?

Let us remember that we assumed earlier that all eigenvalues of matrix $A_{-1}$ are also simple. Starting then from distinct non-zero eigenvalues $\mu_{m}, j=1, \ldots, n$, we are looking for and operator $\mathcal{A}$ with simple eigenvalues only. These eigenvalues can be numbered as $\left\{\lambda_{k}^{m}\right\} \cup\left\{\lambda_{j}^{0}\right\}, m, j=1, \ldots, n ; k \in \mathbb{Z}$, where (see [19, Theorem 1]) the sequence $\left\{\lambda_{k}^{m}\right\}$ satisfies

$$
\sum_{k, m}\left|\lambda_{k}^{m}-\widetilde{\lambda}_{k}^{m}\right|^{2}<\infty, \quad \widetilde{\lambda}_{k}^{m}=\ln \left|\mu_{m}\right|+\mathrm{i}\left(\operatorname{Arg} \mu_{m}+2 \pi k\right) .
$$

Denote by $\left\{\varphi_{k}^{m}\right\} \cup\left\{\varphi_{j}^{0}\right\}, m, j=1, \ldots, n ; k \in \mathbb{Z}$, the corresponding eigenvectors of $\mathcal{A}$. Then (see [19, Lemma 13, Theorem 15]) these vectors form a Riesz basis in $M_{2}$ that is quadratically close to the basis $\left\{\widetilde{\varphi}_{k}^{m}\right\} \cup\left\{\widetilde{\varphi}_{j}^{0}\right\}$ if we assume that the corresponding elements have the same norm. Eigenvector $\{\varphi\}$ has the form

$$
\varphi=\left(\begin{array}{c}
v-\mathrm{e}^{-\lambda} A_{-1} v \\
\mathrm{e}^{\lambda \theta} v
\end{array}\right)
$$


with $\Delta(\lambda) v=0$. Therefore, the fact that the bases $\{\varphi\}$ and $\{\widetilde{\varphi}\}$ are quadratically close implies the condition

$$
\sum_{k}\left\|v_{k}^{m}-y_{m}\right\|^{2}<\infty, \quad m=1, \ldots, n
$$

where $\Delta\left(\lambda_{k}^{m}\right) v_{k}^{m}=0, m=1, \ldots, n ; k \in \mathbb{Z}$, and $y_{m}$ are eigenvectors of $A_{-1}$ corresponding to $\mu_{m}$ as in (4.1). If we apply the same arguments for the dual system

$$
\dot{z}(t)=A_{-1}^{*} \dot{z}(t-1)+\int_{-1}^{0} A_{2}^{*}(\theta) \dot{z}(t+\theta) \mathrm{d} \theta+\int_{-1}^{0} A_{3}^{*}(\theta) z(t+\theta) \mathrm{d} \theta
$$

then we obtain the symmetric condition

$$
\sum_{k}\left\|w_{k}^{m}-z_{m}\right\|^{2}<\infty, \quad m=1, \ldots, n
$$

where $\left(w_{k}^{m}\right)^{*} \Delta\left(\lambda_{k}^{m}\right)=0, m=1, \ldots, n ; k \in \mathbb{Z}$.

Our further goal is to show that conditions (5.1) and (5.4) or (5.1) and (5.2) are almost sufficient for couples of sequences $\{\lambda\}$ and $\{w\}$ or $\{\lambda\}$ and $\{v\}$ to be spectral ones for the system (1.1).

5.1. An operator form for the spectral equation. Let us consider a sequence of pairwise distinct complex numbers $\left\{\lambda_{k_{0}}^{m_{0}}\right\}, m_{0}=1, \ldots, n ; k_{0} \in \mathbb{Z}$, satisfying (5.1). We also assume that the index numbering of $\{\lambda\}$ is such that if $\lambda_{k_{0}}^{m_{0}}=\widetilde{\lambda}_{k}^{m}$ then $m_{0}=m, k_{0}=k$. To begin with, however, we put $\lambda_{k_{0}}^{m_{0}} \neq \widetilde{\lambda}_{k}^{m}$, for all $k, k_{0} \in \mathbb{Z}, m, m_{0}=1, \ldots, n$.

Now let $\left\{\lambda_{k_{0}}^{m_{0}}\right\}$ be simple eigenvalues of operator $\mathcal{A}=\widetilde{\mathcal{A}}+\mathcal{B}_{0} \mathcal{P}_{0}$, where $\mathcal{P}_{0}$ is given by (2.1), in which matrix $A_{3}(\theta)$ satisfies (4.7). Then $\Delta\left(\lambda_{k_{0}}^{m_{0}}\right)=0$ and let $\left\{w_{k_{0}}^{m_{0}}\right\}$ be a sequence of the left degenerating vectors of $\Delta\left(\lambda_{k_{0}}^{m_{0}}\right)$, i.e.

$$
\left(w_{k_{0}}^{m_{0}}\right)^{*} \Delta\left(\lambda_{k_{0}}^{m_{0}}\right)=0
$$

We assume that the sequence $w_{k_{0}}^{m_{0}}$ satisfies (5.4). For all indices $m_{0}=1, \ldots, n$; $k_{0} \in \mathbb{Z}$, consider decompositions

$$
\left(w_{k_{0}}^{m_{0}}\right)^{*}=\sum_{j=1}^{n} \alpha_{j m_{0}}^{k_{0}} z_{j}^{*}
$$

Then condition (5.4) is equivalent to

$$
\sum_{k_{0}}\left|\alpha_{m m_{0}}^{k_{0}}\right|^{2}<\infty, \quad m \neq m_{0}, \sum_{k_{0}}\left|\alpha_{m m}^{k_{0}}-1\right|^{2}<\infty, \quad m, m_{0}=1, \ldots, n .
$$

Let us now rewrite relations (4.5) for $\lambda_{0}=\lambda_{k_{0}}^{m_{0}}$ and $w_{0}=w_{k_{0}}^{m_{0}}$.

We consider the space $\ell_{2}$ of infinite sequences (columns) indexed as $\left\{a_{k}\right\}_{k \in \mathbb{Z}}$ with a scalar product defined by $\left\langle\left\{a_{k}\right\},\left\{b_{k}\right\}\right\rangle=\sum_{k} a_{k} \overline{b_{k}}$. From the relation (4.4), we obtain that vectors

$$
p_{m}^{j}=-\left\{\frac{\bar{p}_{k, m}^{j}}{\widetilde{\widetilde{\beta}}_{k}^{m}}\right\}_{k \in \mathbb{Z}, j, m=1, \ldots, n}
$$


belong to $\ell_{2}$. One can also easily see that

$$
\left\{\frac{1}{\widetilde{\lambda}_{k}^{m}-\lambda_{k_{0}}^{m_{0}}}\right\}_{k \in \mathbb{Z}} \in \ell_{2}, \quad m, m_{0}=1, \ldots, n ; k_{0} \in \mathbb{Z} .
$$

Then, putting $\lambda_{0}=\lambda_{k_{0}}^{m_{0}}$ and $w_{0}=w_{k_{0}}^{m_{0}}$ in the equations (4.5), we obtain

$$
\alpha_{m m_{0}}^{k_{0}}=\sum_{j=1}^{n} \alpha_{j m_{0}}^{k_{0}}\left\langle\left\{\frac{1}{\widetilde{\lambda}_{k}^{m}-\lambda_{k_{0}}^{m_{0}}}\right\}, p_{m}^{j}\right\rangle, \quad m, m_{0}=1, \ldots, n ; k_{0} \in \mathbb{Z} .
$$

We would now like to rewrite relation (5.6) in a vector-matrix abstract form. In order to do that, we introduce a more convenient notation. Denote

$$
\alpha_{m m_{0}}=\left\{\alpha_{m m_{0}}^{k_{0}}\right\}_{k_{0} \in \mathbb{Z}}, \quad m, m_{0}=1, \ldots, n .
$$

By $S_{m m_{0}}=\left\{s_{k_{0} k}^{m m_{0}}\right\}_{k, k_{0} \in \mathbb{Z}}, \quad m, m_{0}=1, \ldots, n$, we denote infinite matrices with elements

$$
s_{k_{0} k}^{m m_{0}}=\frac{1}{\widetilde{\lambda}_{k}^{m}-\lambda_{k_{0}}^{m_{0}}},
$$

and by $A_{j m_{0}}, j, m_{0}=1, \ldots, n$, infinite diagonal matrices

$$
A_{j m_{0}}=\operatorname{diag}\left\{\alpha_{j m_{0}}^{k_{0}}\right\}_{k_{0} \in \mathbb{Z}} .
$$

With these notations, relations (5.6) can be rewritten as

$$
\sum_{j=1}^{n} A_{j m_{0}} S_{m m_{0}} p_{m}^{j}=\alpha_{m m_{0}}
$$

$m, m_{0}=1, \ldots, n$. Now let us fix the index $m$ and consider $n$ equations (5.7) with this index and $m_{0}=1,2, \ldots, n$. Consider another infinite diagonal matrix

$$
\Lambda_{m}=\operatorname{diag}\left\{\widetilde{\lambda}_{k}^{m}-\lambda_{k}^{m}\right\}_{k \in \mathbb{Z}},
$$

and multiply both sides of the $m$-th equality (5.7) (for $m_{0}=m$ ) by this matrix from the left. This gives the following system of equalities

$$
\left\{\begin{array}{l}
\sum_{j=1}^{n} A_{j m_{0}} S_{m m_{0}} p_{m}^{j}=\alpha_{m m_{0}}, \quad m_{0}=1, \ldots, n, m_{0} \neq m, \\
\sum_{j=1}^{n} A_{j m} \Lambda_{m} S_{m m} p_{m}^{j}=\Lambda_{m} \alpha_{m m},
\end{array}\right.
$$

where we use the fact that diagonal matrices commute: $\Lambda_{m} A_{j m}=A_{j m} \Lambda_{m}$. Finally, we introduce block matrix operators

$$
D_{m}=\left[\begin{array}{ccccc}
A_{11} S_{m 1} & \ldots & A_{m 1} S_{m 1} & \ldots & A_{n 1} S_{m 1} \\
\vdots & \ddots & \vdots & & \vdots \\
A_{1 m} \Lambda_{m} S_{m m} & \ldots & A_{m m} \Lambda_{m} S_{m m} & \ldots & A_{n m} \Lambda_{m} S_{m m} \\
\vdots & & \vdots & \ddots & \vdots \\
A_{1 n} S_{m n} & \ldots & A_{m n} S_{m n} & \ldots & A_{n n} S_{m n}
\end{array}\right], m=1, \ldots, n
$$


and present the equations (5.8) in the form

$$
D_{m}\left[\begin{array}{c}
p_{m}^{1} \\
\vdots \\
p_{m}^{m} \\
\vdots \\
p_{m}^{n}
\end{array}\right]=\left[\begin{array}{c}
\alpha_{m 1} \\
\vdots \\
\Lambda_{m} \alpha_{m m} \\
\vdots \\
\alpha_{m n}
\end{array}\right]
$$

Let us observe that both vectors $\left(p_{m}^{1}, \ldots, p_{m}^{n}\right)^{T}$ and $\left(\alpha_{m 1} \ldots \Lambda_{m} \alpha_{m m} \ldots \alpha_{m n}\right)^{T}$ belong to $\ell_{2}^{n}=\underbrace{\ell_{2} \times \ell_{2} \times \ldots \times \ell_{2}}_{n \text { times }}($ see $(4.4),(5.5))$. Therefore the system (5.8) is solvable if and only if the vector $\left(\alpha_{m 1}, \ldots, \Lambda_{m} \alpha_{m m}, \ldots, \alpha_{m n}\right)^{T}$ belongs to the image of operator $D_{m}$ as an operator from $\ell_{2}^{n}$ to $\ell_{2}^{n}$. In the following, we show that, for all sequences $\{\lambda\}$ satisfying (5.1), and for almost all sequences $\{w\}$ satisfying (5.4), operators $D_{m}, m=1, \ldots, n$, are bounded and have bounded inverse operators from $\ell_{2}^{n}$ to $\ell_{2}^{n}$. This means that the spectral assignment problem is solvable.

5.2. Characterization of the spectral assignment. In what follows, we need a proposition, that characterizes a Riesz basis in $L_{2}(0,1)$.

Proposition 5.1. Let $\left\{\lambda_{k}\right\}_{k \in \mathbb{Z}}$ be a sequence such that

$$
\sum_{k \in \mathbb{Z}}\left|\lambda_{k}-a+\mathrm{i}(b+2 \pi k)\right|^{2}<\infty
$$

for some $a, b \in \mathbb{R}$. Then the family $\left\{\mathrm{e}^{\lambda_{k} t}, k \in \mathbb{Z}\right\}$ forms a Riesz basis in $L_{2}(0,1)$.

There are several ways to prove this classic result (see [1]). It may be obtained, for example, from the Paley-Wiener theorem [13] and Lemma II.4.11 [1].

Next we prove the following preliminary result.

LEMma 5.2. 1. For $m \neq m_{0}$ linear operators $S_{m m_{0}}: \ell_{2} \rightarrow \ell_{2}$ are bounded and have bounded inverses. 2. The linear operator $\Lambda_{m} S_{m m}: \ell_{2} \rightarrow \ell_{2}$ is a bounded operator and has a bounded inverse.

Proof. Let $\left\{\varphi_{k}\right\},\left\{\widetilde{\varphi}_{k}\right\}, k \in \mathbb{Z}$, be two Riesz bases of a Hilbert space $H$ and let $R$ be a bounded operator with a bounded inverse, such that $R \varphi_{k}=\widetilde{\varphi}_{k}, k \in \mathbb{Z}$.

For $f \in H$, we have

$$
f=\sum_{j} a_{j} \varphi_{j}, \quad R f=\sum_{j} a_{j} R \varphi_{j}
$$

Then

$$
R \varphi_{j}=\widetilde{\varphi}_{j}=\sum_{k} c_{j k} \varphi_{k}=\sum_{k}\left\langle\widetilde{\varphi}_{j}, \psi_{k}\right\rangle \varphi_{k}, \quad j \in \mathbb{Z},
$$

where $\left\{\psi_{k}\right\}_{k \in \mathbb{Z}}$ is the bi-orthogonal with respect to the basis $\left\{\varphi_{k}\right\}_{k \in \mathbb{Z}}$. Hence

$$
R f=\sum_{j} a_{j} \sum_{k}\left\langle\widetilde{\varphi}_{j}, \psi_{k}\right\rangle \varphi_{k}=\sum_{k} \sum_{j} a_{j}\left\langle\widetilde{\varphi}_{j}, \psi_{k}\right\rangle \varphi_{k}=\sum_{k} b_{k} \varphi_{k}
$$

where $b_{k}=\sum_{j} a_{j}\left\langle\widetilde{\varphi}_{j}, \psi_{k}\right\rangle$.

This means that the infinite matrix $\widehat{R}$ corresponding to $R$ in the basis $\left\{\varphi_{k}\right\}$ is of the form

$$
\widehat{R}=\left\{\widehat{r}_{k j}=\left\langle\widetilde{\varphi}_{j}, \psi_{k}\right\rangle\right\}_{\substack{k \in \mathbb{Z} \\ j \in \mathbb{Z}}},
$$


where

$$
\widehat{R}\left[\begin{array}{c}
\vdots \\
a_{-1} \\
a_{0} \\
a_{1} \\
\vdots
\end{array}\right]=\left[\begin{array}{c}
\vdots \\
b_{-1} \\
b_{0} \\
b_{1} \\
\vdots
\end{array}\right],\left\{a_{j}\right\},\left\{b_{j}\right\} \in \ell_{2} .
$$

Let now $H=L_{2}(0,1)$ and $\left\{\widetilde{\varphi}_{k}\right\}_{k \in \mathbb{Z}}$ be a Riesz basis of the form $\widetilde{\varphi}_{k}=\mathrm{e}^{\widetilde{\lambda}_{k}^{m} t}$ for some $m=1, \ldots, n$. Let now $\left\{\varphi_{k}\right\}_{k \in \mathbb{Z}}$ be a Riesz basis that is bi-orthogonal to $\left\{\psi_{k}=\mathrm{e}^{-\overline{\lambda_{k}^{m}} t}\right\}_{k \in \mathbb{Z}}$ for some $m_{0}=1, \ldots, n$ (the fact that $\left\{\psi_{k}\right\}_{k \in \mathbb{Z}}$ is a Riesz basis of $L_{2}(0,1)$ follows from Proposition 5.1). One has

$$
\begin{aligned}
\left\langle\widetilde{\varphi}_{j}, \psi_{k}\right\rangle & =\int_{-1}^{0} \mathrm{e}^{\widetilde{\lambda}_{j}^{m} t} \mathrm{e}^{-\lambda_{k}^{m_{0}} t} d t=\frac{1}{\widetilde{\lambda}_{j}^{m}-\lambda_{k}^{m_{0}}}\left(\mathrm{e}^{\widetilde{\mathrm{j}}_{j}^{m}-\lambda_{k}^{m_{0}}}-1\right) \\
& =\frac{1}{\widetilde{\lambda}_{j}^{m}-\lambda_{k}^{m_{0}}}\left(\mu_{m} \mathrm{e}^{-\lambda_{k}^{m_{0}}}-1\right),
\end{aligned}
$$

i.e. $\widehat{R}=\left\{\widehat{r}_{k j}\right\}_{\substack{k \in \mathbb{Z} \\ j \in \mathbb{Z}}}$ where

$$
\widehat{r}_{k j}=s_{k j}\left(\mu_{m} \mathrm{e}^{-\lambda_{k}^{m_{0}}}-1\right), \quad k, j \in \mathbb{Z} .
$$

Thus

$$
\widehat{R}=\mathcal{E}_{m m_{0}} S_{m m_{0}}
$$

where $\mathcal{E}_{m m_{0}}$ is the infinite matrix

$$
\mathcal{E}_{m m_{0}}=\left[\begin{array}{ccccc}
\ddots & \ldots & \ldots & \ldots & \ldots \\
\vdots & \mu_{m} \mathrm{e}^{-\lambda_{-1}^{m_{0}}-1} & 0 & 0 & \vdots \\
\vdots & 0 & \mu_{m} \mathrm{e}^{-\lambda_{0}^{m_{0}}-1} & 0 & \vdots \\
\vdots & 0 & 0 & \mu_{m} \mathrm{e}^{-\lambda_{1}^{m_{0}}}-1 & \vdots \\
\ldots & \ldots & \ldots & \ldots & \ddots
\end{array}\right] .
$$

Hence, we have the following alternative:

1. If $m_{0} \neq m$, then the sequence $\left\{\mu_{m} \mathrm{e}^{-\lambda_{k}^{m_{0}}}-1\right\}_{k \in \mathbb{Z}}$ is bounded and separated from 0, i.e. $\mathcal{E}_{m m_{0}}: \ell_{2} \rightarrow \ell_{2}$ is a bounded operator with a bounded inverse. Hence,

$$
S_{m m_{0}}=\mathcal{E}_{m m_{0}}^{-1} \hat{R} \text {. }
$$

2. If $m=m_{0}$, then $\mu_{m} \mathrm{e}^{-\lambda_{k}^{m}} \rightarrow 1, k \rightarrow \infty$. Moreover,

$$
\begin{aligned}
\mu_{m} \mathrm{e}^{-\lambda_{k}^{m}}-1 & =\mathrm{e}^{\widetilde{\lambda}_{k}^{m}-\lambda_{k}^{m}}-1 \\
& =\left(1+\left(\widetilde{\lambda}_{k}^{m}-\lambda_{k}^{m}\right)+\ldots+\frac{\left(\widetilde{\lambda}_{k}^{m}-\lambda_{k}^{m}\right)^{s}}{s !}+\ldots\right)-1 \\
& =\left(\widetilde{\lambda}_{k}^{m}-\lambda_{k}^{m}\right)+o\left(\widetilde{\lambda}_{k}^{m}-\lambda_{k}^{m}\right) .
\end{aligned}
$$


Therefore,

$$
\mathcal{E}_{m m}=\Lambda_{m} Q_{m}=Q_{m} \Lambda_{m},
$$

where $Q_{m}=\operatorname{diag}\left(1+O\left(\widetilde{\lambda}_{k}^{m}-\lambda_{k}^{m}\right)\right)_{k \in \mathbb{Z}}$ has a bounded inverse, so

$$
\Lambda_{m} S_{m m}=Q_{m}^{-1} \hat{R} .
$$

From (5.9) and (5.10), it follows that $S_{m m_{0}}, m \neq m_{0}$ and $\Lambda_{m} S_{m m}$ are bounded and have bounded inverses.

REMARK 5.3. In our previous consideration we assumed implicitly that our sequences $\left\{\lambda_{k_{0}}^{m_{0}}\right\}_{k_{0} \in \mathbb{Z}}$ are different from $\left\{\widetilde{\lambda}_{k}^{m}\right\}_{k \in \mathbb{Z}}$, i.e. $\tilde{\lambda}_{k}^{m} \neq \lambda_{k_{0}}^{m_{0}}$ for all $k, k_{0} \in$ $\mathbb{Z}, m, m_{0} \in\{1, \ldots, n\}$ in particular $\lambda_{k}^{m} \neq \widetilde{\lambda}_{k}^{m}$ for every $k \in \mathbb{Z}, m \in\{1, \ldots, n\}$.

Now let us allow $\lambda_{k}^{m}=\widetilde{\lambda}_{k}^{m}$ for some indices $k \in I \subset \mathbb{Z}$. Note that, in this case, the operators $S_{m m_{0}}, m_{0} \neq m$, are still well-defined and the operator $\Lambda_{m} S_{m m}$ can also be well-defined if we define its components as limits of the corresponding components when $\lambda_{k}^{m} \rightarrow \widetilde{\lambda}_{k}^{m}, k \in I$. This means that for $k \in I$ all non-diagonal elements of the $k$-th line of $\Lambda_{m} S_{m m}$ equal 0 and the diagonal elements equal 1. Besides, $S_{m m_{0}}$ for $m_{0} \neq m$ and $\Lambda_{m} S_{m m}$ remain bounded with bounded inverse operators since formulas (5.9) and (5.10) also remain true when $\lambda_{k}^{m}=\widetilde{\lambda}_{k}^{m}$. Finally, if we consider the dependence $\Lambda_{m} S_{m m}$ of the sequence $\left\{\lambda_{k}^{m}\right\}_{k \in \mathbb{Z}}$, one can easily prove that

$$
\Lambda_{m} S_{m m}\left(\left\{\lambda_{k}^{\prime m}\right\}\right) \rightarrow \Lambda_{m} S_{m m}\left(\left\{\lambda_{k}^{m}\right\}\right)
$$

as

$$
\sum_{k}\left|\lambda_{k}^{\prime m}-\lambda_{k}^{m}\right|^{2} \rightarrow 0
$$

In other words, the operators $\Lambda_{m} S_{m m}$ and consequently their inverse $\left(\Lambda_{m} S_{m m}\right)^{-1}$, depend continuously on sequences $\left\{\lambda_{k}^{m}\right\}_{k \in \mathbb{Z}}$ of the set

$$
\left\{\left\{\lambda_{k}^{m}\right\}: \sum_{k}\left|\lambda_{k}^{m}-\tilde{\lambda}_{k}^{m}\right|^{2}<\infty\right\} .
$$

Now we are ready to prove our main results on the spectral assignment.

TheOREM 5.4. Let $\mu_{1}, \mu_{2}, \ldots, \mu_{n}$ be different non-zero complex numbers and $z_{1}, z_{2}, \ldots, z_{n}$ be non-zero $n$-dimensional linear independent vectors. Denote

$$
\tilde{\lambda}_{k}^{m}=\ln \left|\mu_{m}\right|+\mathrm{i}\left(\operatorname{Arg} \mu_{m}+2 \pi k\right), \quad m=1, \ldots, n, k \in \mathbb{Z} .
$$

Let us consider an arbitrary sequence of different complex numbers $\left\{\lambda_{k}^{m}\right\}_{m=1, \ldots, n}^{k \in \mathbb{Z}}$ such that

$$
\sum_{k}\left|\lambda_{k}^{m}-\widetilde{\lambda}_{k}^{m}\right|^{2}<\infty, \quad m=1, \ldots, n .
$$

Then there is a small enough $\varepsilon>0$ such that for any sequence of non-zero vectors $\left\{d_{k}^{m}\right\}_{\substack{k \in \mathbb{Z} \\ m=1, \ldots, n}}$ satisfying

$$
\sum_{k}\left\|d_{k}^{m}-z_{m}\right\|^{2}<\varepsilon, \quad m=1, \ldots, n
$$


one can choose matrices $A_{-1}, A_{2}(\theta), A_{3}(\theta)$ such that for the system (1.1), with these matrices, the following two conditions hold:

i) all the numbers $\left\{\lambda_{k}^{m}\right\}$ are roots of the characteristic equation $\operatorname{det} \Delta(\lambda)=0$, $k \in \mathbb{Z}, m=1, \ldots, n$,

ii) $d_{k}^{m}$ are right degenerating vectors for the matrix $\Delta\left(\lambda_{k}^{m}\right): d_{k}^{m *} \Delta\left(\lambda_{k}^{m}\right)=$ $0, m=1, \ldots, n ; k \in \mathbb{Z}$.

Such a choice is unique if we put the following additional condition on the matrix $A_{3}(\theta)$ :

$$
\int_{-1}^{0} A_{3}(\theta) \mathrm{d} \theta=0
$$

Proof. First, we denote by $A_{-1}$ the matrix uniquely defined by the relations:

$$
z_{m}^{*} A_{-1}=\mu_{m} z_{m}^{*}, m=1, \ldots, n,
$$

and denote by $\left\{y_{j}\right\}_{j=1, \ldots, n}$ the bi-orthogonal basis with respect to $\left\{z_{j}\right\}_{j=1, \ldots, n}$ in $\mathbb{C}^{n}$. Then the corresponding operator $\widetilde{\mathcal{A}}$, generated by the matrix $A_{-1}$, has eigenvalues $\widetilde{\lambda}_{k}^{m}, m=1, \ldots, n, k \in \mathbb{Z} ; \widetilde{\lambda}_{0}=0$ and possesses a Riesz basis of eigenvectors of $\left\{\widetilde{\varphi}_{k}^{m}\right\}_{k \in \mathbb{Z}} \cup\left\{\widetilde{\varphi}_{j}^{0}\right\}_{j=1, \ldots, n}$. In the case when all $\mu_{m} \neq 1, m=1, \ldots, n$, the corresponding eigenvectors of $\widetilde{\mathcal{A}}$ are

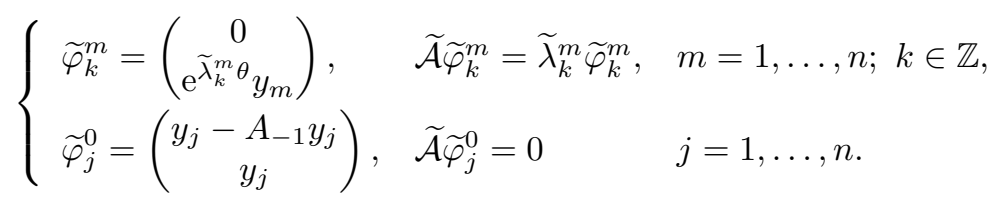

In the case $\mu_{1}=1$, the vector

$$
\varphi_{0}^{1}=\left(\begin{array}{c}
A_{-1} y_{j} \\
\theta y_{j}
\end{array}\right), \quad j=1, \ldots, n,
$$

is a generalized eigenvector of $\widetilde{\mathcal{A}}$ corresponding to $\widetilde{\lambda}_{0}$ and the other $\widetilde{\varphi}_{k}^{m}, \widetilde{\varphi}_{j}^{0}$ are given by formula (5.11). Let us show that there is a choice of a bounded operator $\mathcal{P}_{0}: X_{\widetilde{\mathcal{A}}} \rightarrow \mathbb{C}^{n}$ (or equivalently a choice of matrices $A_{2}(\theta), A_{3}(\theta)$ ) such that

$$
\lambda_{k_{0}}^{m_{0}} \in \sigma\left(\widetilde{\mathcal{A}}+\mathcal{B}_{0} \mathcal{P}_{0}\right),
$$

or equivalently $\operatorname{det} \Delta\left(\lambda_{k_{0}}^{m_{0}}\right)=0$, and

$$
d_{k_{0}}^{m_{0} *} \Delta\left(\lambda_{k_{0}}^{m_{0}}\right)=0, \quad m=1, \ldots, n ; k \in \mathbb{Z} .
$$

We represent vectors $d_{k_{0}}^{m_{0}}$ in the basis $\left\{z_{j}\right\}_{j=1, \ldots, n}$, namely

$$
d_{k_{0}}^{m_{0}}=\sum_{m=1}^{n} \alpha_{m m_{0}}^{k_{0}} z_{m}, \quad k_{0} \in \mathbb{Z} ; m, m_{0}=1, \ldots, n .
$$

With these notations the condition

$$
\sum_{k_{0}}\left\|d_{k_{0}}^{m}-z_{m}\right\|^{2}<\infty, \quad m=1, \ldots, n
$$


implies

$$
\sum_{k_{0}}\left|\alpha_{m m_{0}}^{k_{0}}\right|^{2}<\infty, \quad m \neq m_{0} ; \quad \sum_{k_{0}}\left|\alpha_{m m}^{k_{0}}-1\right|^{2}<\infty, m=1, \ldots, n .
$$

and these sums tend to zero as

$$
\sum_{k_{0}}\left\|d_{k_{0}}^{m}-z_{m}\right\|^{2} \rightarrow 0
$$

Therefore, under condition (5.14), the operators $D_{m}, m=1, \ldots, n$ tend to the blockdiagonal operators

$$
\widehat{D}_{m}=\left[\begin{array}{cccccc}
S_{m 1} & 0 & \ldots & 0 & \ldots & 0 \\
\vdots & \vdots & \vdots & \vdots & & \vdots \\
0 & 0 & \ldots & \Lambda_{m} S_{m m} & \ldots & 0 \\
\vdots & \vdots & \vdots & \vdots & \vdots & \vdots \\
0 & 0 & \ldots & 0 & \ldots & S_{m n}
\end{array}\right]
$$

which have a bounded inverse due to Lemma 5.2. This means that for a small enough $\varepsilon>0$, the inequality $\sum_{k}\left\|d_{k}^{m}-z_{m}\right\|^{2}<\varepsilon$ implies that operators $D_{m}, m=1, \ldots, n$ have bounded inverses. If numbers $\left\{\lambda_{k}^{m}\right\}$ are different from $\left\{\tilde{\lambda}_{k}^{m}\right\}$, this means that there is a bounded operator $\mathcal{P}_{0}: X_{\mathcal{A}} \rightarrow \mathbb{C}^{n}$ for which the relations (5.12) and (5.13) are satisfied. Besides, this operator is unique if we also require the additional assumption: $\mathcal{P}_{0} \widetilde{\varphi}_{j}^{0}=0, j=1, \ldots, n$, which is equivalent to condition (C) of the Theorem. If we allow coincidences $\lambda_{k}^{m}=\widetilde{\lambda}_{k}^{m}$ for some indices $\{k, m\} \in \mathbb{I}$, we need to use the continuous dependence of operators $D_{m}$ on the sequence $\left\{\lambda_{k}^{m}\right\}$ (see Remark 5.3). We approximate $\left\{\lambda_{k}^{\prime m}\right\}$ by $\left\{\lambda_{k}^{\prime m}\right\}\left(\sum_{k}\left|\lambda_{k}^{\prime m}-\lambda_{k}^{m}\right|^{2} \rightarrow 0\right)$ such that $\left\{\lambda_{k}^{\prime m}\right\} \neq \widetilde{\lambda}_{k}^{m}$. Since the conditions (5.12) and (5.13) are satisfied for the operator $\mathcal{P}_{0}\left(\left\{\lambda_{k}^{\prime m}\right\}\right)$ they are also satisfied for $\mathcal{P}_{0}\left(\left\{\lambda_{k}^{m}\right\}\right)$. This completes the proof.

LEMMA 5.5. Let $\left\{\lambda_{k}^{m}\right\}$ be a given sequence such that $\sum_{k}\left|\lambda_{k}^{m}-\widetilde{\lambda}_{k}^{m}\right|^{2}<\infty$ and $\left\{\widehat{\alpha}_{m m_{0}}^{k_{0}}\right\}$ is an arbitrary sequence satisfying

$$
\sum_{k_{0}}\left|\widehat{\alpha}_{m m_{0}}^{k_{0}}\right|^{2}<\infty, \quad m \neq m_{0} ; \quad \sum_{k_{0}}\left|\widehat{\alpha}_{m m}^{k_{0}}-1\right|^{2}<\infty .
$$

Then for any $\varepsilon>0$ and $m=1, \ldots, n$ there is a sequence $\left\{\alpha_{m m_{0}}^{k_{0}}\right\}$ satisfying

$$
\sum_{k_{0}}\left|\alpha_{m m_{0}}^{k_{0}}-\widehat{\alpha}_{m m_{0}}^{k_{0}}\right|^{2}<\varepsilon
$$

and such that the operator $D_{m}$ has a bounded inverse.

Proof. First, for a given $\left\{\lambda_{k}^{m}\right\}$, let us choose $\varepsilon_{0}>0$ such that $D_{m}$ will be invertible for

$$
\sum_{k_{0}}\left|\alpha_{m m_{0}}^{k_{0}}\right|^{2}<\varepsilon_{0}, \quad m \neq m_{0}, \quad \sum_{k_{0}}\left|\alpha_{m m}^{k_{0}}-1\right|^{2}<\varepsilon_{0} .
$$


Then, one can find a large enough $N$ such that

$$
\sum_{\left|k_{0}\right|>N}\left|\widehat{\alpha}_{m m_{0}}^{k_{0}}\right|^{2}<\varepsilon_{0}, \quad \sum_{\left|k_{0}\right|>N}\left|\widehat{\alpha}_{m m}^{k_{0}}-1\right|^{2}<\varepsilon_{0}
$$

Next we consider the sequences $\left\{\alpha_{m m_{0}}^{k_{0}}\right\}$ for which

$$
\alpha_{m m_{0}}^{k_{0}}=\widehat{\alpha}_{m m_{0}}^{k_{0}}, \quad\left|k_{0}\right|>N
$$

Our goal is to choose the remaining components $\alpha_{m m_{0}}^{k_{0}},\left|k_{0}\right| \leq N$ in order to satisfy the requirement of our lemma. Denote rows of matrix $D_{m}$ by $\left(\ell_{k_{0}}^{m_{0}}\right)^{*}, m_{0}=$ $1, \ldots, n ; k_{0} \in \mathbb{Z}$ and let $q_{k_{0}}^{m_{0}}$ be the corresponding components of the vector $q=D_{m} p$, i.e. $q_{k_{0}}^{m_{0}}=\left(\ell_{k_{0}}^{m_{0}}\right)^{*} p, p \in \ell_{2}^{n}, m_{0}=1, \ldots, n ; k_{0} \in \mathbb{Z}$. The space $L=\underbrace{\ell_{2} \times \ldots \times \ell_{2}}_{n \text { times }}$ may be written as $L=L^{1} \oplus L^{2}$, where

$$
L^{1}=\left\{q: q_{k_{0}}^{m_{0}}=0, m_{0}=1, \ldots, n,\left|k_{0}\right|>N\right\},
$$

and

$$
L^{2}=\left\{q: q_{k_{0}}^{m_{0}}=0, m_{0}=1, \ldots, n,\left|k_{0}\right| \leq N\right\} .
$$

Let $P$ be the orthogonal projector on $L^{2}$. Let us observe that the rows $\left(\ell_{k_{0}}^{m_{0}}\right)^{*}$ for $\left|k_{0}\right|>N$ do not depend on components $\alpha_{m m_{0}}^{k_{0}},\left|k_{0}\right| \leq N$ and that, if we put $\alpha_{m m_{0}}^{k_{0}}=\delta_{m m_{0}},\left|k_{0}\right| \leq N, m, m_{0}=1, \ldots, n$, then the operator $D_{m}: L \rightarrow L$ has a bounded inverse. This means that, for all sequences $\left\{\alpha_{m m_{0}}^{k_{0}}\right\}$ satisfying (5.15), we have $P D_{m} L=L^{2}$ and the invertibility of $D_{m}$ occurs if and only if

$$
D_{m} L \supset L^{1} \text {. }
$$

Let $L^{1^{\prime}}$ be the subspace

$$
\left\{p \in L:\left(\ell_{k_{0}}^{m_{0}}\right)^{*} p=0,\left|k_{0}\right|>N, m_{0}=1, \ldots, n\right\}
$$

of dimension $(2 N+1) n$. Denote by $\ell_{k}^{j^{\prime}}, j=1, \ldots, n ;|k| \leq N$ a basis of $L^{1^{\prime}}$. With these notations, one can see that $(5.16)$ is equivalent to the invertibility of $(2 N+$ 1) $n \times(2 N+1) n$ matrix

$$
M=\left\{\left(\ell_{k_{0}}^{m_{0}}\right)^{*} \ell_{k}^{j^{\prime}},\left|k_{0}\right| \leq N,|k| \leq N, m_{0}=1, \ldots, n, j=1, \ldots, n\right\}
$$

i.e. $\operatorname{det} M \neq 0$. The components of $M$ are linear functions of $\alpha_{m m_{0}}^{k_{0}},\left|k_{0}\right| \leq N$. Therefore, the determinant $\operatorname{det} M$ is a polynomial whose coefficients are also linear functions of these scalars. Besides, $\operatorname{det} M$ is not identical to zero because the matrix $D_{m}$ is invertible if we take $\alpha_{m m_{0}}^{k_{0}}=\delta_{m m_{0}}$, i.e. $\operatorname{det} M \neq 0$. This implies that $M$ is invertible almost everywhere in $\mathbb{C}^{2(N+1) n}$. This fact completes the proof of Lemma 5.5 because we can choose $\alpha_{m m_{0}}^{k_{0}},\left|k_{0}\right| \leq N$, in such a way that

$$
\sum_{\left|k_{0}\right| \leq N}\left|\alpha_{m m_{0}}^{k_{0}}-\widehat{\alpha}_{m m_{0}}^{k_{0}}\right|<\varepsilon
$$

and the operator $D_{m}$ will be invertible. 
REMARK 5.6. From the proof of Lemma 5.5, it is easy to see that the sequence $\alpha_{m m_{0}}^{k_{0}}$ may differ from $\widehat{\alpha}_{m m_{0}}^{k_{0}}$ only for a finite number of components $\left|k_{0}\right| \leq N$.

Due to Lemma 5.5, the formulation of Theorem 5.4 may be generalized in the following way.

TheOREM 5.7. Let the sequence $\left\{\lambda_{k}^{m}\right\}_{k \in \mathbb{Z}, m=1, \ldots, n}$ and vectors $z_{m}, m=1, \ldots, n$ be chosen according to the assumptions of Theorem 5.4. Then, for any sequence of vectors $\left\{\widehat{d}_{k}^{m}\right\}_{k \in \mathbb{Z}, m=1, \ldots, n}$ satisfying

$$
\sum_{k}\left\|\widehat{d}_{k}^{m}-z_{m}\right\|^{2}<\infty, m=1, \ldots, n
$$

and for any $\varepsilon>0$, there is a sequence $\left\{d_{k}^{m}\right\}_{k \in \mathbb{Z}, m=1, \ldots, n}$ :

$$
\sum_{k}\left\|d_{k}^{m}-\widehat{d}_{k}^{m}\right\|^{2}<\varepsilon
$$

such that, for some choice of matrices $A_{-1}, A_{2}(\theta), A_{3}(\theta)$, satisfying $\int_{-1}^{0} A_{3}(\theta) \mathrm{d} \theta=0$, the conditions i) and ii) of Theorem 5.4 are verified. Moreover, $\left\{d_{k}^{m}\right\}$ may be chosen in such a way, that $d_{k}^{m}=\widehat{d}_{k}^{m}$ for all $|k|>N$ and for some $N \in \mathbb{N}$.

Now we can prove the final result on the spectral assignment.

THEOREM 5.8. Let $\mu_{1}, \mu_{2}, \ldots, \mu_{n}$ be different non-zero complex numbers and $z_{1}, z_{2}, \ldots, z_{n}$ be non-zero $n$-dimensional linear independent vectors. Denote

$$
\tilde{\lambda}_{k}^{m}=\ln \left|\mu_{m}\right|+\mathrm{i}\left(\operatorname{Arg} \mu_{m}+2 \pi k\right), \quad m=1, \ldots, n, k \in \mathbb{Z} .
$$

Let us consider an arbitrary sequence of different complex numbers $\left\{\lambda_{k}^{m}\right\}_{\substack{k \in \mathbb{Z} \\ m=1, \ldots, n}}$ such that

$$
\sum_{k}\left|\lambda_{k}^{m}-\widetilde{\lambda}_{k}^{m}\right|^{2}<\infty, \quad m=1, \ldots, n
$$

Let $\left\{\widehat{d}_{k}^{m}\right\}_{k \in \mathbb{Z}, m=1, \ldots, n}$ be quadratically close to the sequences $\left\{z_{m}\right\}_{m=1, \ldots, n}$ :

$$
\sum_{k}\left\|\widehat{d}_{k}^{m}-z_{m}\right\|^{2}<\infty, m=1, \ldots, n
$$

In addition, let complex numbers $\lambda_{j}^{0}, j=1, \ldots, n$ be different from each other and different from $\lambda_{k}^{m}$ and let $d_{j}^{0}, j=1, \ldots, n$ be linear independent vectors.

Then, for any $\varepsilon>0$, there exists $N>0$ and a sequence $\left\{d_{k}^{m}\right\}_{k \in \mathbb{Z}, m=1, \ldots, n}$ :

$$
d_{k}^{m}=\widehat{d}_{k}^{m}, \quad|k|>N, \quad \sum_{|k| \leq N}\left\|d_{k}^{m}-\widehat{d}_{k}^{m}\right\|^{2}<\varepsilon, m=1, \ldots, n
$$

such that there are some matrices $A_{-1}, A_{2}(\theta), A_{3}(\theta)$ for which the following conditions are satisfied:

i) The set $\left\{\lambda_{k}^{m}\right\}_{k \in \mathbb{Z}, m=1, \ldots, n} \cup\left\{\lambda_{j}^{0}\right\}_{j=1 \ldots, n}$ is the set of all roots of the characteristic equation $\operatorname{det} \Delta(\lambda)=0$;

ii) $d_{k}^{m^{*}} \Delta\left(\lambda_{k}^{m}\right)=0, m=1, \ldots, n, k \in \mathbb{Z}$ and $d_{j}^{0^{*}} \Delta\left(\lambda_{j}^{0}\right)=0$. 
Proof. Denote by $C$ a $(n \times n)$ matrix uniquely defined by the equalities:

$$
d_{j}^{0^{*}} C=\lambda_{j}^{0} d_{j}^{0^{*}}, j=1, \ldots, n,
$$

and let us put, for any $k \in \mathbb{Z}, m=1, \ldots, n$,

$$
\widehat{f}_{k}^{m^{*}}=\left\{\begin{array}{lll}
\widehat{d}_{k}^{m^{*}}\left(I-\frac{1}{\lambda_{k}^{m}} C\right)^{-1} & \text { if } & \lambda_{k}^{m} \neq 0, \\
\widehat{d}_{k}^{m^{*}} C^{-1} & \text { if } & \lambda_{k}^{m}=0 .
\end{array}\right.
$$

It is easy to see that the sequences $\left\{\widehat{f}_{k}^{m}\right\}$ are also quadratically close to $z_{m}$ :

$$
\sum_{k}\left\|\widehat{f}_{k}^{m}-z_{m}\right\|^{2}<\infty
$$

Therefore, due to Theorem 5.7, for any $\varepsilon>0$, there are matrices $A_{-1}, \widehat{A}_{2}(\theta), \widehat{A}_{3}(\theta)$ $\left(\int_{-1}^{0} \widehat{A}_{3}(\theta) \mathrm{d} \theta=0\right)$, a number $N>0$ and a sequence of vectors $\left\{f_{k}^{m}\right\}_{k \in \mathbb{Z}, m=1, \ldots, n}$ verifying

$$
\sum_{k}\left\|f_{k}^{m}-\widehat{f}_{k}^{m}\right\|^{2}<\frac{\varepsilon}{M^{2}}, \quad f_{k}^{m}=\widehat{f}_{k}^{m},|k|>N
$$

such that

$$
f_{k}^{m *} \widehat{\Delta}\left(\lambda_{k}^{m}\right)=0, k \in \mathbb{Z}, \quad m=1, \ldots, n,
$$

where $M=\sup \left\{\left\|I-\frac{1}{\lambda_{k}^{m}} C\right\|, \lambda_{k}^{m} \neq 0 ;\|C\|\right\}$ and

$$
\widehat{\Delta}(\lambda)=\lambda I-\lambda \mathrm{e}^{-\lambda} A_{-1}+\int_{-1}^{0} \lambda \mathrm{e}^{\lambda \theta} \widehat{A}_{2}(\theta) \mathrm{d} \theta+\int_{-1}^{0} \mathrm{e}^{\lambda \theta} \widehat{A}_{3}(\theta) \mathrm{d} \theta .
$$

We have $\widehat{\Delta}(0)=0$, and the function $\widehat{\Delta}_{1}(\lambda)=\frac{1}{\lambda} \widehat{\Delta}(\lambda)$ may be extended to $\lambda=0$ by the formula

$$
\widehat{\Delta}_{1}(0)=I-A_{-1}+\int_{-1}^{0} \widehat{A}_{2}(\theta) \mathrm{d} \theta+\int_{-1}^{0} \lim _{\lambda \rightarrow 0} \frac{\mathrm{e}^{\lambda \theta}-1}{\lambda} \widehat{A}_{3}(\theta) \mathrm{d} \theta .
$$

Now, let us put

$$
\Delta(\lambda)=\left(I-\frac{1}{\lambda} C\right) \widehat{\Delta}(\lambda), \quad \lambda \neq 0 .
$$

At $\lambda=0$, the function $\Delta(\lambda)$ may be defined as

$$
\Delta(0)=-C \widehat{\Delta}_{1}(0) .
$$

We can observe that $\Delta(\lambda)$ can be written as

$$
\begin{aligned}
\Delta(\lambda)= & \lambda I-\lambda \mathrm{e}^{-\lambda} A_{-1}+\int_{-1}^{0} \lambda \mathrm{e}^{\lambda \theta} \widehat{A}_{2}(\theta) \mathrm{d} \theta+\int_{-1}^{0} \mathrm{e}^{\lambda \theta} \widehat{A}_{3}(\theta) \mathrm{d} \theta \\
& -C+\mathrm{e}^{-\lambda} C A_{-1}-\int_{-1}^{0} \mathrm{e}^{\lambda \theta} C \widehat{A}_{2}(\theta) \mathrm{d} \theta+\int_{-1}^{0} \mathrm{e}^{\lambda \theta} \int_{-1}^{\theta} C \widehat{A}_{3}(\tau) \mathrm{d} \tau \mathrm{d} \theta, \\
= & \lambda I-\lambda \mathrm{e}^{-\lambda} A_{-1}+\int_{-1}^{0} \lambda \mathrm{e}^{\lambda \theta} A_{2}(\theta) \mathrm{d} \theta+\int_{-1}^{0} \mathrm{e}^{\lambda \theta} A_{3}(\theta) \mathrm{d} \theta,
\end{aligned}
$$


for $A_{2}(\theta)$ and $A_{3}(\theta)$ given by

$$
\begin{aligned}
& A_{2}(\theta)=\widehat{A}_{2}(\theta)-(\theta+1) C-\theta C A_{-1}, \\
& A_{3}(\theta)=\widehat{A}_{3}(\theta)+\int_{-1}^{\theta} C \widehat{A}_{3}(\tau) \mathrm{d} \tau-C-C A_{-1}
\end{aligned}
$$

It is now easy to see that, with this choice of matrices, the conditions i) and ii) are satisfied. Indeed,

$$
\operatorname{det} \Delta(\lambda)=\operatorname{det}\left(I-\frac{1}{\lambda} C\right) \operatorname{det} \widehat{\Delta}(\lambda)
$$

and then, the numbers $\left\{\lambda_{k}^{m}\right\}$ and $\{\lambda\}_{j}^{0}$ are roots of the characteristic equation and

$$
\begin{aligned}
& d_{k}^{m * \Delta}\left(\lambda_{k}^{m}\right)=f_{k}^{m *} \widehat{\Delta}\left(\lambda_{k}^{m}\right)=0, \quad k \in \mathbb{Z}, m=1, \ldots, n, \\
& d_{j}^{0 *} \Delta\left(\lambda_{j}^{0}\right)=0, \quad j=1, \ldots, m .
\end{aligned}
$$

Finally, for all $m=1, \ldots, n$, we have

$$
\sum_{k}\left\|d_{k}^{m}-\widehat{d}_{k}^{m}\right\|^{2} \leq \sum_{k, m} M^{2}\left\|f_{k}^{m}-\widehat{f}_{k}^{m}\right\|<\varepsilon .
$$

This completes the proof. $\square$

EXAMPLE 5.9. We consider equations of the form

$$
\left(\begin{array}{c}
\dot{z}_{1}(t) \\
\dot{z}_{2}(t)
\end{array}\right)=\left(\begin{array}{c}
z_{1}(t-1) \\
-z_{2}(t-1)
\end{array}\right)+\int_{-1}^{\theta}\left(A_{2}(\theta)\left(\begin{array}{c}
\dot{z}_{1}(t+\theta) \\
\dot{z}_{2}(t+\theta)
\end{array}\right)+A_{3}(\theta)\left(\begin{array}{c}
z_{1}(t+\theta) \\
z_{2}(t+\theta)
\end{array}\right)\right) \mathrm{d} \theta
$$

where $A_{l}(\theta)=\left(a_{i j}^{l}\right)_{i, j=1,2}, l=2,3$. For this case matrix $A_{-1}$ equals

$$
A_{-1}=\left(\begin{array}{cc}
1 & 0 \\
0 & -1
\end{array}\right)
$$

and then $\mu_{1}=1, \mu_{2}=-1$. Hence $\tilde{\lambda}_{k}^{1}=2 \pi k \mathrm{i}, \tilde{\lambda}_{k}^{2}=\pi \mathrm{i}+2 \pi k \mathrm{i}, z_{1}=\left(\begin{array}{l}1 \\ 0\end{array}\right), z_{2}=\left(\begin{array}{l}0 \\ 1\end{array}\right)$. Let us put $\lambda_{k}^{m}=\tilde{\lambda}_{k}^{m}, k \in \mathbb{Z}, m=1,2$, and let us choose for arbitrary $N \in \mathbb{N}$ the sequence $\left\{d_{k}^{m}\right\}, k \in \mathbb{Z}, m=1,2$, with $d_{k}^{m}=z_{m}$ for $|k|>N, m=1,2$, and $d_{k}^{m}=z_{2}$ for $|k| \leq N$, $m=1,2$. This sequence is quadratically close to $z_{m}$, i.e.

$$
\sum_{k \in \mathbb{Z}}\left\|d_{k}^{m}-z_{m}\right\|^{2}<\infty, \quad m=1,2 .
$$

If we try to find $A_{2}(\theta), A_{3}(\theta)$ corresponding to $\left\{\lambda_{k}^{m}\right\},\left\{d_{k}^{m}\right\}$, then this leads to the equalities

$$
\int_{-1}^{0}(\pi+2 \pi k) \mathrm{ie}^{(\pi+2 \pi k) \mathrm{i} \theta} a_{22}^{2}(\theta) d \theta+\int_{-1}^{0} \mathrm{e}^{(\pi+2 \pi k) \mathrm{i} \theta} a_{22}^{3}(\theta) d \theta=0, \quad k \in \mathbb{Z},
$$

and

$$
\int_{-1}^{0} 2 \pi k \mathrm{ie}{ }^{2 \pi k \mathrm{i} \theta} a_{22}^{2}(\theta) d \theta+\int_{-1}^{0} \mathrm{e}^{2 \pi k \mathrm{i} \theta} a_{22}^{3}(\theta) \mathrm{d} \theta=4 \pi k \mathrm{i}, \quad|k| \leq N .
$$


This in turn can be rewritten as

$$
\begin{aligned}
& \int_{-1}^{0} \mathrm{e}^{(\pi+2 \pi k) \mathrm{i} \theta}\left(a_{22}^{2}(\theta)-\int_{0}^{\theta} a_{22}^{3}(\tau) \mathrm{d} \tau\right) \mathrm{d} \theta=\frac{\mathrm{i} C}{(\pi+2 \pi k)}, \quad k \in \mathbb{Z}, \\
& \int_{-1}^{0} \mathrm{e}^{2 \pi k \mathrm{i} \theta}\left(a_{22}^{2}(\theta)-\int_{0}^{\theta} a_{22}^{3}(\tau) d \tau\right) d \theta=2+\frac{\mathrm{i} C}{2 \pi k}, \quad|k| \leq N,
\end{aligned}
$$

where $C=\int_{-1}^{0} a_{22}^{3}(\theta) \mathrm{d} \theta$. From (5.17) one has

$$
a_{22}^{2}(\theta)-\int_{0}^{\theta} a_{22}^{3}(\tau) d \tau=\sum_{k \in \mathbb{Z}} \mathrm{e}^{(-\pi+2 \pi k) \mathrm{i} \theta} \frac{\mathrm{i} C}{(\pi+2 \pi k)}
$$

which obviously contradicts (5.18). This means that our choice of $d_{k}^{m}$ is impossible. However, if we slightly change vectors $d_{k}^{m},|k| \leq N$, as $d_{k}^{m}=z_{2}+\sqrt{\frac{\varepsilon}{2 N+1}} z_{1}$ for arbitrarily small $\varepsilon>0$, and put

$$
\begin{aligned}
a_{11}^{3}(\theta) & =2 \sqrt{\frac{2 N+1}{\varepsilon}} \sum_{k=-N}^{N} \mathrm{e}^{-2 \pi k \mathrm{i} \theta} \\
& =2 \sqrt{\frac{2 N+1}{\varepsilon}} \frac{\mathrm{e}^{2(2 N+1) \pi \mathrm{i} \theta}-1}{\mathrm{e}^{2 \pi \mathrm{i} \theta}-1} \cdot \mathrm{e}^{-2 \pi N \mathrm{i} \theta},
\end{aligned}
$$

and

$$
a_{21}^{3}(\theta)=a_{12}^{3}(\theta)=a_{22}^{3}(\theta)=0, \quad a_{j s}^{2}(\theta)=0, \quad j, s=1,2,
$$

one can verify that

$$
d_{k}^{m *} \Delta\left(\lambda_{k}^{m}\right)=0, \quad k \in \mathbb{Z}, \quad m=1,2 .
$$

Besides,

$$
\sum_{k}\left\|d_{k}^{m}-z_{m}\right\|^{2}=\varepsilon
$$

Finally, note that $\left\|a_{11}^{3}(\cdot)\right\| \rightarrow \infty$ as $N \rightarrow \infty$ or $\varepsilon \rightarrow 0$. Thus, such a choice of eigenvalues is possible only for a finite number of elements.

6. The moment problem and the controllability. Consider the control neutral-type system given by

$$
\dot{z}(t)=A_{-1} \dot{z}(t-1)+\int_{-1}^{0} A_{2}(\theta) \dot{z}(t+\theta) d \theta+\int_{-1}^{0} A_{3}(\theta) z(t+\theta) d \theta+B u(t),
$$

where the matrices $A_{-1}, A_{2}(\cdot), A_{3}(\cdot)$ are as in (1.1), $B$ is an $n \times r$-matrix, and $u(t)$ is an $r$-vector control. In the function space $M_{2}$, this system may be written as

$$
\frac{\mathrm{d}}{\mathrm{d} t}\left(\begin{array}{c}
y(t) \\
z_{t}(\cdot)
\end{array}\right)=\mathcal{A}\left(\begin{array}{c}
y(t) \\
z_{t}(\cdot)
\end{array}\right)+\mathcal{B} u
$$


where the operator $\mathcal{B}: \mathbb{R}^{r} \rightarrow M_{2}$ is defined by $\mathcal{B} u=(B u, 0)^{T}$.

As in the preceding sections, we consider the case when all eigenvalues of $A_{-1}$ are simple. Denote these eigenvalues by $\mu_{m}, m=1, \ldots, n$. The operator $\widetilde{\mathcal{A}}$, corresponding to the case $A_{2}(\theta)=A_{3}(\theta)=0$, has simple eigenvalues

$$
\tilde{\lambda}_{k}^{m}=\ln \left|\mu_{m}\right|+\mathrm{i}\left(\operatorname{Arg} \mu_{m}+2 \pi k\right), m=1, \ldots, n, k \in \mathbb{Z},
$$

with corresponding eigenvectors $\widetilde{\varphi}_{k}^{m}$. The other eigenvalue of $\widetilde{\mathcal{A}}$ is $\widetilde{\lambda}^{0}=0$, which corresponds to an $n$-dimensional subspace generated by the generalized eigenvectors: $\widetilde{\varphi}_{1}^{0}, \ldots, \widetilde{\varphi}_{n}^{0}$. All eigenvectors $\left\{\widetilde{\varphi}_{k}^{m}\right\} \cup\left\{\widetilde{\varphi}_{j}^{0}\right\}$ constitute a Riesz basis in $M_{2}$.

We assume, as in the preceding sections, that $A_{2}(\theta)$ and $A_{3}(\theta)$ are chosen in such a way that all eigenvalues of $\mathcal{A}, \lambda_{k}^{m}, m=1, \ldots, n, k \in \mathbb{Z}$, are simple. As noted before, they satisfy the condition $[18,19]$ :

$$
\sum_{k, m}\left|\lambda_{k}^{m}-\widetilde{\lambda}_{k}^{m}\right|^{2}<\infty
$$

and the corresponding eigenvectors $\left\{\varphi_{k}^{m}\right\}$ satisfy

$$
\sum_{k, m}\left\|\varphi_{k}^{m}-\widetilde{\varphi}_{k}^{m}\right\|^{2}<\infty .
$$

Eigenvectors $\left\{\varphi_{k}^{m}\right\}$ together with a set of generalized eigenvectors $\left\{\varphi_{j}^{0}, j=1, \ldots, n\right\}$ form a Riesz basis in $M_{2}$ quadratically close to the spectral basis $\{\widetilde{\varphi}\}$ of $\widetilde{\mathcal{A}}$.

Let $\{\widetilde{\psi}\}$ and $\{\psi\}$ be biorthogonal bases for $\{\widetilde{\varphi}\}$ and $\{\varphi\}$ respectively. It is known that $\{\widetilde{\psi}\}$ and $\{\psi\}$ are spectral bases of adjoint operators $\widetilde{\mathcal{A}}^{*}$ and $\mathcal{A}^{*}$,

$$
\widetilde{\mathcal{A}}^{*} \widetilde{\psi}_{k}^{m}=\overline{\bar{\lambda}}_{k}^{m} \widetilde{\psi}_{k}^{m}, \quad \mathcal{A}^{*} \psi_{k}^{m}=\bar{\lambda}_{k}^{m} \psi_{k}^{m} .
$$

These bases are also quadratically close [5]:

$$
\sum_{k, m}\left\|\psi_{k}^{m}-\widetilde{\psi}_{k}^{m}\right\|^{2}<\infty
$$

Finally, let us note that, as shown in [20], vectors $\widetilde{\psi}_{k}^{m}$, and $\psi_{k}^{m}, k \neq 0$, are of the form

$$
\widetilde{\psi}_{k}^{m}=\left(\begin{array}{c}
\frac{1}{k+\frac{1}{2}} z_{m} \\
*
\end{array}\right), \quad \psi_{k}^{m}=\left(\begin{array}{c}
\frac{1}{k+\frac{1}{2}} d_{k}^{m} \\
*
\end{array}\right),
$$

where $z_{m}, m=1, \ldots, n$ are eigenvectors of $A_{-1}^{*}, A_{-1}^{*} z_{m}=\bar{\mu}_{m} z_{m}$, and the $n$-vectors $d_{k}^{m}$ are such that $\Delta^{*}\left(\bar{\lambda}_{k}^{m}\right) d_{k}^{m}=0$, and

$$
\sum_{k, m}\left\|d_{k}^{m}-z_{m}\right\|^{2}<\infty, \quad m=1, \ldots, n .
$$

Now, we use the biorthogonal bases $\{\varphi\}$ and $\{\psi\}$ to interpret the steering conditions of the controllability problem of the system (6.1). Let $x \in M_{2}$. Then

$$
x=\sum_{\varphi \in\{\varphi\}}\langle x, \psi\rangle \cdot \varphi .
$$


A state $x=\left(\begin{array}{c}y \\ z(\cdot)\end{array}\right) \in M_{2}$ is reachable at time $T$ by a control $u(\cdot) \in L_{2}\left(0, T ; \mathbb{C}^{r}\right)$ if and only if the steering condition

$$
x=\left(\begin{array}{c}
y \\
z(\cdot)
\end{array}\right)=\int_{0}^{T} \mathrm{e}^{\mathcal{A} t} \mathcal{B} u(t) \mathrm{d} t .
$$

holds. This relation may be expanded using the basis $\{\varphi\}$. A state $x$ is reachable if and only

$$
\sum_{\varphi \in\{\varphi\}}\langle x, \psi\rangle \cdot \varphi=\sum_{\varphi \in\{\varphi\}} \int_{0}^{T}\left\langle\mathrm{e}^{\mathcal{A} t} \mathcal{B} u(t), \psi\right\rangle \mathrm{d} t \cdot \varphi,
$$

for some $u(\cdot) \in L_{2}\left(-1,0 ; \mathbb{R}^{r}\right)$. Then, the steering condition (6.6) can be substituted by the following system of equalities

$$
\langle x, \psi\rangle=\int_{0}^{T}\left\langle\mathrm{e}^{\mathcal{A} t} \mathcal{B} u(t), \psi\right\rangle \mathrm{d} t, \quad \psi \in\{\psi\} .
$$

Let $\left\{b_{1}, \ldots, b_{r}\right\}$ be columns of $B$ and $\boldsymbol{b}_{i}=\left(\begin{array}{c}b_{i} \\ 0\end{array}\right) \in M_{2}, i=1, \ldots, r$. Then, the right hand side of (6.7) takes the form

$$
\int_{0}^{T}\left\langle\mathrm{e}^{\mathcal{A} t} \mathcal{B} u(t), \psi\right\rangle \mathrm{d} t=\sum_{i=1}^{r} \int_{0}^{T}\left\langle\mathrm{e}^{\mathcal{A} t} \boldsymbol{b}_{i}, \psi\right\rangle u_{i}(t) \mathrm{d} t .
$$

Taking into account the fact that $\{\psi\}$ is a spectral basis of $\mathcal{A}^{*}$, we can rewrite the main steering conditions, corresponding to the basis $\left\{\psi_{k}^{m}\right\}$ as

$$
s_{k}^{m}=\left(k+\frac{1}{2}\right)\left\langle x, \psi_{k}^{m}\right\rangle=\int_{0}^{T} \mathrm{e}^{\lambda_{k}^{m} t}\left(b_{k, m}^{1} u_{1}(t)+\ldots+b_{k, m}^{r} u_{r}(t)\right) \mathrm{d} t,
$$

$m=1, \ldots, n, k \in \mathbb{Z}$, where

$$
b_{k, m}^{j}=\left(k+\frac{1}{2}\right)\left\langle\boldsymbol{b}_{j}, \psi_{k}^{m}\right\rangle=\left\langle\left(\begin{array}{c}
b_{j} \\
0
\end{array}\right),\left(\begin{array}{c}
d_{k}^{m} \\
*
\end{array}\right)\right\rangle .
$$

Equations (6.8) pose a vector moment problem with respect to unknown functions $u_{j}(t), j=1, \ldots, r$. Using (6.4), we observe that

$$
\left(k+\frac{1}{2}\right)\left\langle\boldsymbol{b}_{j}, \widetilde{\psi}_{k}^{m}\right\rangle=\left\langle\left(\begin{array}{c}
b_{j} \\
0
\end{array}\right),\left(\begin{array}{c}
z_{m} \\
*
\end{array}\right)\right\rangle=\left\langle b_{j}, z_{m}\right\rangle=b_{m}^{j},
$$

Thus, this value does not depend on $k$. Then from (6.5) it follows that the coefficients $b_{k, m}^{j}$ satisfy the condition

$$
\sum_{k, m}\left|b_{k, m}^{j}-b_{m}^{j}\right|^{2}=\sum_{k, m}\left|\left\langle b_{j}, d_{k}^{m}-z_{m}\right\rangle\right|^{2}<\infty \quad j=1, \ldots, r .
$$

In the scalar case $r=1$, under the conditions

$$
b_{m}^{1}=\left\langle\boldsymbol{b}_{1}, \psi_{m}\right\rangle \neq 0, \quad m=1, \ldots, n
$$


(controllability of the pair $\left.\left(A_{-1}, B\right)\right)$ and

$$
\left\langle\boldsymbol{b}_{1}, \psi_{k}^{m}\right\rangle \neq 0, \quad m=1, \ldots, n ; k \in \mathbb{Z}
$$

(approximate controllability of system (6.2)), the solvability of (6.8) can be studied by application of the methods given in [1]. In the vector case, the problem is more complicated. We showed [16] that the minimal interval of solvability of (6.2) is related to the first controllability index $n_{1}\left(A_{-1}, B\right)$ of the system $\dot{x}=A_{-1} x+B u$ (see [27]).

Our purpose now is to consider the abstract moment problems of the form (6.8) by means of their correspondence to the controllability problems for neutral-type systems (6.1).

We show first that the conditions (6.3) and (6.9), together with some conditions analogous to (6.10) and (6.11), are not only necessary but also almost sufficient for the moment problem (6.8) to be generated by a neutral type system (6.1).

THEOREM 6.1. Let us consider the moment problem (6.8). Let the following conditions be satisfied:

i) $\sum_{k, m}\left|\lambda_{k}^{m}-\widetilde{\lambda}_{k}^{m}\right|^{2}<\infty$, where $\tilde{\lambda}_{k}^{m}=\ln \left|\mu_{m}\right|+\mathrm{i}\left(\operatorname{Arg} \mu_{m}+2 \pi k\right)$,

$m=1, \ldots, n, k \in \mathbb{Z}, \mu_{1}, \ldots, \mu_{r}$ are some non-zero different complex numbers;

ii) $\sum_{j, k, m}\left|b_{k, m}^{j}-b_{m}^{j}\right|^{2}<\infty$, where $b_{m}^{j}$ and $b_{k, m}^{j}$ are some real numbers such that

$$
\sum_{j=1}^{r}\left|b_{m}^{j}\right|>0, \quad \sum_{j=1}^{r}\left|b_{k, m}^{j}\right|>0, \quad m=1, \ldots, n ; k \in \mathbb{Z} .
$$

Then, for any $\varepsilon>0$, there is an integer $N>0$, and sequences of scalars $\left\{b_{k, m}^{\prime j}\right\}$, $m=1, \ldots, n ; j=1, \ldots, r$ :

$$
b_{k, m}^{\prime j}=b_{k, m}^{j}, \quad|k|>N, \quad \sum_{|k| \leq N}\left|b_{k, m}^{\prime j}-b_{k, m}^{j}\right|^{2}<\varepsilon,
$$

and matrices $A_{-1}, A_{2}(\cdot), A_{2}(\cdot), B$ such that the control system (6.1) with these matrices is exactly controllable and the steering conditions for this system will correspond to the moment problem

$$
s_{k}^{m}=\int_{0}^{T} \mathrm{e}^{\lambda_{k}^{m} t}\left(b^{\prime 1}{ }_{k, m} u_{1}(t)+\ldots+b_{k, m}^{\prime r} u_{r}(t)\right) \mathrm{d} t .
$$

Proof. Let us introduce the $n \times n$ and $n \times r$-matrices $A_{-1}$ and $B$ given by

$$
A_{-1}=\operatorname{diag}\left\{\mu_{1}, \ldots, \mu_{n}\right\}, \quad B=\left\{b_{m}^{j}\right\}_{m=1, \ldots, n}^{j=1, \ldots, r} .
$$

The basis of eigenvectors $\left\{z_{m}\right\}$ of the matrix $A_{-1}^{*}$ is the canonical basis:

$$
z_{m}=e_{m}=(0, \ldots, 1, \ldots, 0)^{*}
$$

Due to conditions ii) we have

$$
z_{m}^{*} B=\left(b_{m}^{1}, \ldots, b_{m}^{r}\right) \neq 0 .
$$

This means that the pair $\left(A_{-1}, B\right)$ is controllable. 
Now we use Theorem 5.8. Let us choose a sequence of vectors $\left\{\widehat{d}_{k}^{m}\right\}$ satisfying the conditions

$$
\left\langle b_{j}, \widehat{d}_{k}^{m}\right\rangle=b_{k, m}^{j}, \quad k \in \mathbb{Z} ; m=1, \ldots, n ; j=1, \ldots, r
$$

and

$$
\sum_{k \in \mathbb{Z}}\left\|\widehat{d}_{k}^{m}-z_{m}\right\|^{2}<\infty
$$

From conditions ii) of the theorem, we obtain

$$
\inf _{k, m} \sum_{j=1}^{r}\left|b_{k, m}^{j}\right|=\omega>0 .
$$

Therefore we can choose $\varepsilon_{0}>0$ sufficiently small, that

$$
\sum_{j=1}^{r}\left\|\left\langle b_{j}, d_{k}^{m}\right\rangle\right\| \neq 0, \quad k \in \mathbb{Z} ; m=1, \ldots, n,
$$

as $\left\|d_{k}^{m}-\widehat{d}_{k}^{m}\right\|<\varepsilon_{0}$.

Applying Theorem 5.8 we observe that, for any $\varepsilon>0$, there are $N>0$ and matrices $A_{2}(\cdot), A_{3}(\cdot)$ such that the corresponding operator $\mathcal{A}$ will have the eigenvalues $\left\{\lambda_{k}^{m}\right\}$ with the related eigenvectors $d_{k}^{m}$ :

$$
d_{k}^{m *} \Delta\left(\lambda_{k}^{m}\right)=0, \quad k \in \mathbb{Z} ; m=1, \ldots, n,
$$

where

$$
d_{k}^{m}=\widehat{d}_{k}^{m}, \quad|k|>N ; \quad \sum_{|k|<N}\left\|\widehat{d}_{k}^{m}-d_{k}^{m}\right\|<\frac{\varepsilon}{M}, \quad M=\max _{j=1, \ldots, r}\left\|b_{j}\right\| .
$$

Let us now denote

$$
b_{k, m}^{\prime j}=\left\langle b_{j}, d_{k}^{m}\right\rangle
$$

Then it is easy to see that

$$
\left|b_{k, m}^{j}-b_{k, m}^{\prime j}\right|=\left|\left\langle b_{j}, \widehat{d}_{k}^{m}\right\rangle\right|<\varepsilon, \quad k \in \mathbb{Z} ; m=1, \ldots, n ; j=1, \ldots, r .
$$

If we assume that $\varepsilon<M \varepsilon_{0}$, then we obtain from (6.15) that

$$
\sum_{j=1}^{r}\left|b_{k, m}^{\prime j}\right|>0, \quad k \in \mathbb{Z} ; m=1, \ldots, n ; j=1, \ldots, r .
$$

The last relation may be formulated as

$$
\operatorname{rank}\left(\Delta\left(\lambda_{k}^{m}\right), B\right)=n, \quad k \in \mathbb{Z} ; m=1, \ldots, n,
$$

which is equivalent to the approximate controllability property of the system (6.1). This condition with the controllability property of the pair $\left(A_{-1}, B\right)$ gives $[16]$ the exact controllability of the system (6.1). Thus, the moment problem (6.12) corresponds to the steering conditions of the constructed exactly controllable system (6.1). 
Let us now analyze the solvability of the moment problem (6.8). Let $S_{T}, T>0$ be the linear operator that associates the function $u(\cdot) \in L_{2}\left([0, T] ; \mathbb{R}^{r}\right)$ with the sequence $\left\{s_{k}^{m}\right\}$ :

$$
S_{T} u(\cdot)=\left\{s_{k}^{m}\right\}
$$

Denote by $I_{T}$ the image of the operator $S_{T}$. First we can observe that the Riesz basis property of exponentials $\left\{\mathrm{e}^{\lambda_{k}^{m} t}\right\}$ (see Proposition 5.1) implies $I_{T} \subset \ell_{2}$. Moreover $S_{T}$ is bounded and it is easy to see that

$$
I_{T_{1}} \subset I_{T_{2}}, \quad \text { if } \quad T_{1}<T_{2} .
$$

This makes the following definition natural.

DEFINITION 6.2. 1. The moment problem is completely solvable if there is $T>0$ such that $I_{T}=\ell_{2}$. 2. For a completely solvable moment problem, the interval $\left[0, T^{*}\right]$, where $T^{*}=\inf \left\{T: I_{T}=\ell_{2}\right\}$ is called the critical interval of solvability for the moment problem.

When a moment problem corresponds to the controllability problem of an exactly controllable neutral-type system of the form (6.1), it is known from [16] that the length of the critical interval of solvability can be calculated by the formula

$$
T^{*}=n_{1}\left(A_{-1}, B\right)=\min \left\{k: \operatorname{rank}\left(B, A_{-1} B, \ldots, A_{-1}^{k-1} B\right)=n\right\} .
$$

The integer $n_{1}\left(A_{-1}, B\right)$ is the first of a set of controllability indices for the pair $\left(A_{-1}, B\right)$ (see for example [27]). Our goal is to prove the same relation for the abstract moment problem (6.8) satisfying the assumptions of Theorem 6.1.

LEMMA 6.3. Let for some $T_{0}>0$ and $\varepsilon_{0}>0$ the image not increase in the interval $\left.] T_{0}, T_{0}+\varepsilon\right]$, i.e.

$$
I_{T_{1}}=I_{T_{2}}, \quad T_{0}<I_{T_{1}} \leq I_{T_{2}} \leq T_{0}+\varepsilon
$$

Then this relation holds for all $T_{0}<I_{T_{1}} \leq I_{T_{2}}<\infty$.

Proof. For any $\varepsilon>0$ we introduce the operator $E_{\varepsilon}: \ell_{2} \rightarrow \ell_{2}$ by

$$
E_{\varepsilon}\left\{s_{k}^{m}\right\}=\left\{\mathrm{e}^{\lambda_{k}^{m} \varepsilon} s_{k}^{m}\right\} .
$$

Let us observe that if $\left\{s_{k}^{m}\right\}=S_{T} u(\cdot)$, then

$$
E_{\varepsilon}\left\{s_{k}^{m}\right\}=\left\{\int_{0}^{T} \mathrm{e}^{\lambda_{k}^{m}(t+\varepsilon)} \sum_{j=1}^{r} b_{k, m}^{j} u_{j}(t) \mathrm{d} t\right\}=\left\{\int_{\varepsilon}^{T+\varepsilon} \mathrm{e}^{\lambda_{k}^{m} \tau} \sum_{j=1}^{r} b_{k, m}^{j} u_{j}(\tau-\varepsilon) \mathrm{d} \tau\right\},
$$

and then $E_{\varepsilon} S_{T} u(\cdot) \in I_{T+\varepsilon}$. This relation together with (6.16) brings the inclusions

$$
E_{\varepsilon} I_{T_{1}} \subset I_{T_{1}+\varepsilon}=I_{T_{0}+\varepsilon_{0}}=I_{T_{1}}
$$

for all $T_{0}<T_{1}<T_{1}+\varepsilon \leq T_{0}+\varepsilon$. Then, for any $N \in \mathbb{N}$, we obtain

$$
E_{N \varepsilon} I_{T_{1}}=E_{(N-1) \varepsilon}\left(E_{\varepsilon} I_{T_{1}}\right) \subset E_{(N-1) \varepsilon}\left(I_{T_{1}}\right) \subset \cdots \subset E_{\varepsilon}\left(I_{T_{1}}\right) \subset I_{T_{1}} .
$$

Hence

$$
I_{T_{1}+N \varepsilon} \subset I_{T_{1}} \cup E_{\varepsilon} I_{T_{1}} \cup \cdots \cup E_{N \varepsilon} I_{T_{1}} \subset I_{T_{1}},
$$


which implies $I_{T_{1}+N \varepsilon}=I_{T_{1}}$. If $N$ is chosen such that $T_{1}+N \varepsilon \geq T_{2}$, then

$$
I_{T_{2}} \subset I_{T_{1}+N \varepsilon}=I_{T_{1}} \subset I_{T_{2}} .
$$

This proves our Lemma. $\square$

THEOREM 6.4. Let the moment problem (6.8) satisfy assumptions i) and ii) of Theorem 6.1. Then this problem is completely solvable and the length of the critical interval of solvability is $T^{*}=n_{1}\left(A_{-1}, B\right)$, where

$$
A_{-1}=\operatorname{diag}\left\{\mu_{1}, \ldots, \mu_{n}\right\}, \quad B=\left\{b_{m}^{j}\right\}_{m=1, \ldots, n}^{j=1, \ldots, r} .
$$

Proof. First we note that, by Theorem 6.1, the moment problem (6.8) almost corresponds (up to the substitution of a finite set $\left\{b_{k, m}^{\prime j}\right\}_{|k| \leq N}$ by $\left\{b_{k, m}^{j}\right\}_{|k| \leq N}$ ) to the steering conditions for some exactly controllable system (6.1) whose minimal time of controllability is $n_{1}\left(A_{-1}, B\right)$. This means, in particular, that for

$$
T>T^{*}=n_{1}\left(A_{-1}, B\right),
$$

the images $I_{T}$ are subspaces of finite co-dimension in the space $\ell_{2}$. Our goal is to prove that $I_{T}=\ell_{2}$, for $T>T^{*}$. Since $\operatorname{codim} I_{T}<\infty$ for $T>T^{*}$, it is sufficient to show that $I_{T}=\ell_{2}$ for some $T>0$.

In parallel with the problem (6.8), let us consider the classic (scalar) moment problem

$$
s_{k}^{m}=\int_{0}^{T} \mathrm{e}^{\lambda_{k}^{m} t} v(t) \mathrm{d} t, \quad k \in \mathbb{Z}, m=1, \ldots, n, \quad v(\cdot) \in L_{2}(0, T) .
$$

It is well known [1] that the family of exponential $\left\{\mathrm{e}^{\lambda_{k}^{m}} t\right\}$ forms a Riesz basis in $L_{2}(0, n)$ and hence the problem is completely solvable for $T \geq n$. We want to show that our problem (6.8) is also solvable for this $T$.

Let us put

$$
u_{j}(t)=\alpha_{j} v(t), \quad j=1, \ldots, r .
$$

For this particular substitution, the relations of our moment problem (6.8) take the form

$$
s_{k}^{m}=\int_{0}^{T} \mathrm{e}^{\lambda_{k}^{m} t}\left(\sum_{j=1}^{r} \alpha_{j} b_{k, m}^{j}\right) v(t) \mathrm{d} t .
$$

Now, let us remark that the scalars $\alpha_{j}, j=1, \ldots, r$ can be chosen in such a way that

$$
\sum_{j=1}^{r} \alpha_{j} b_{k, m}^{j} \neq 0, \quad k \in \mathbb{Z}, m=1, \ldots, n .
$$

Indeed, if not, then the countable family of hyperplanes

$$
\Pi_{k, m}=\left\{\left(\alpha_{1}, \ldots, \alpha_{r}\right) \in \mathbb{R}^{r}: \sum_{j=1}^{r} \alpha_{j} b_{k, m}^{j}=0\right\}, \quad k \in \mathbb{Z}, m=1, \ldots, n
$$


covers the whole space $\mathbb{R}^{r}$. This contradicts the Baire theorem. Moreover, the assumptions ii) of the theorem (see the formulation of Theorem 6.1) imply that the sequence $\left\{b_{k, m}^{j}\right\}$ is bounded and $\cup_{k, m} \Pi_{k, m}$ is a closed set. From these considerations, we can conclude that there are two constants $c_{1}$ and $c_{2}$ such that

$$
0<c_{1}<\sum_{j=1}^{r} \alpha_{j} b_{k, m}^{j}<c_{2}
$$

This means that problems (6.17) and (6.18) have the same set of solvability. Then the problem (6.18) is solvable for all $\left\{s_{k}^{m}\right\} \in \ell_{2}$ if $T>T^{*}$. Since $I_{T}$ includes the set of solvability of (6.18), we obtain that $I_{T}=\ell_{2}$ for all $T>T^{*}$. $\square$

Finally, we give the following illustrative example.

EXAMPLE 6.5. Consider two vector moment problems:

$$
\left\{\begin{array}{l}
s_{k}^{1}=\int_{0}^{T} \mathrm{e}^{\left(\mathrm{i}(\pi+2 \pi k)+\varepsilon_{k}^{1}\right) t}\left(11 u_{1}(t)+u_{2}(t)\right) d t \\
s_{k}^{2}=\int_{0}^{T} \mathrm{e}^{\left(\mathrm{i} 2 \pi k+\varepsilon_{k}^{2}\right) t}\left(u_{1}(t)+u_{2}(t)\right) d t \\
s_{k}^{3}=\int_{0}^{T} \mathrm{e}^{\left(\log 2+\mathrm{i} 2 \pi k+\varepsilon_{k}^{3}\right) t}\left(u_{1}(t)-u_{2}(t)\right) d t \\
s_{k}^{4}=\int_{0}^{T} \mathrm{e}^{\left(\log 3+\mathrm{i} 2 \pi k+\varepsilon_{k}^{4}\right) t}\left(u_{1}(t)-u_{2}(t)\right) d t
\end{array}\right.
$$

and

$$
\left\{\begin{array}{l}
s_{k}^{1}=\int_{0}^{T} \mathrm{e}^{\left(\mathrm{i}(\pi+2 \pi k)+\varepsilon_{k}^{1}\right) t}\left(6 u_{1}(t)-u_{2}(t)\right) d t \\
s_{k}^{2}=\int_{0}^{T} \mathrm{e}^{\left(\mathrm{i} 2 \pi k+\varepsilon_{k}^{2}\right) t}\left(4 u_{1}(t)-u_{2}(t)\right) d t \\
s_{k}^{3}=\int_{0}^{T} \mathrm{e}^{\left(\log 2+\mathrm{i} 2 \pi k+\varepsilon_{k}^{3}\right) t}\left(3 u_{1}(t)-u_{2}(t)\right) d t \\
s_{k}^{4}=\int_{0}^{T} \mathrm{e}^{\left(\log 3+\mathrm{i} 2 \pi k+\varepsilon_{k}^{4}\right) t}\left(2 u_{1}(t)-u_{2}(t)\right) d t
\end{array}\right.
$$

$k \in \mathbb{Z}, \sum_{k}\left(\varepsilon_{k}^{j}\right)^{2}<\infty, j=1, \ldots, 4$. Both moment problems can be reduced to the controllability problem for systems of the form (6.1), where

$$
A_{-1}=\left(\begin{array}{cccc}
0 & 1 & 0 & 0 \\
0 & 0 & 1 & 0 \\
0 & 0 & 0 & 1 \\
6 & -5 & -5 & 5
\end{array}\right)
$$

$\left\{\mu_{1}, \mu_{2}, \mu_{3}, \mu_{4}\right\}=\{-1,1,2,3\}$, and the corresponding eigenvectors of $A_{-1}^{*}$ are

$$
z_{1}=\left(\begin{array}{c}
-6 \\
11 \\
-6 \\
1
\end{array}\right), \quad z_{2}=\left(\begin{array}{c}
6 \\
1 \\
-4 \\
1
\end{array}\right), \quad z_{3}=\left(\begin{array}{c}
3 \\
-1 \\
-3 \\
1
\end{array}\right), \quad z_{4}=\left(\begin{array}{c}
2 \\
-1 \\
-2 \\
1
\end{array}\right)
$$

The control matrices $B_{1}$ and $B_{2}$ for both systems (6.19) and (6.20) are

$$
B_{1}=\left(\begin{array}{ll}
0 & 0 \\
1 & 0 \\
0 & 0 \\
0 & 1
\end{array}\right), \quad B_{2}=\left(\begin{array}{ll}
0 & 0 \\
0 & 0 \\
1 & 0 \\
0 & 1
\end{array}\right)
$$


for a certain choice of $A_{2}(\theta), A_{3}(\theta)$. Since $n_{1}\left(A_{-1}, B_{1}\right)=2$ and $n_{1}\left(A_{-1}, B_{2}\right)=3$, the moment problem (6.19) is solvable for all $\left\{s_{k}^{j}\right\} \in \ell_{2}$ if $T>2$ and the problem (6.20) is solvable if $T>3$.

7. Conclusion. We give here some conditions for sets of complex numbers $\{\lambda\}$ and $n$-vectors $\{d\}$ such that they form a spectral set for a neutral-type system. This is a first step in solving vector moment problems using the exact controllability properties of a neutral-type system related to the given moment problem.

\section{REFERENCES}

[1] S. A. Avdonin And S. A. Ivanov, Families of exponentials. The method of moments in controllability problems for distributed parameter systems, Cambridge University Press, Cambridge, 1995.

[2] R. Bellman And K. L. Cooke, Differential-difference equations, Academic Press, New York, 1963.

[3] J. A. Burns, T. L. Herdman, and H. W. Stech, Linear functional-differential equations as semigroups on product spaces, SIAM J. Math. Anal., 14 (1983), pp. 98-116.

[4] R. F. CURTAin AND H. ZWART, An introduction to infinite-dimensional linear systems theory, vol. 21 of Texts in Applied Mathematics, Springer-Verlag, New York, 1995.

[5] I. C. Gohberg AND M. G. KRein, Introduction to the theory of linear nonselfadjoint operators, Translations of Mathematical Monographs, Vol. 18, American Mathematical Society, Providence, R.I., 1969.

[6] J. K. Hale And S. M. Verduyn Lunel, Introduction to functional-differential equations, vol. 99 of Applied Mathematical Sciences, Springer-Verlag, New York, 1993.

[7] - Strong stabilization of neutral functional differential equations, IMA J. Math. Control Inform., 19 (2002), pp. 5-23.

[8] M. I. KADEC', The exact value of the Paley-Wiener constant, Dokl. Akad. Nauk SSSR, 155 (1964), pp. 1253-1254.

[9] V. B. Kolmanovskil And A. MYshkis, Introduction to the theory and applications of functional-differential equations, Kluwer Academic Publishers, Dordrecht, 1999.

[10] B. JA. LEvin, On Riesz basis of exponentials in $L^{2}$, Zapiski Matematicheskogo Otdeleniya Fiziko-matematicheskogo Fakulteta Kharkovskogo Universiteta, 27 (1961), pp. 39-48.

[11] B. C. Moore, On the flexibility offered by state feedback in multivariable systems beyond closed loop eigenvalue assignment, IEEE Trans. Automatic Control, AC-21 (1976), pp. 689-692.

$[12]$ D. A. O'Connor And T. J. TARn, On stabilization by state feedback for neutral differentialdifference equations, IEEE Trans. Automat. Control, 28 (1983), pp. 615-618.

[13] R. E. A. C. PAley AND N. Wiener, Fourier transforms in the complex domain, vol. 19 of AMS Colloquium Publ., Providence, RI, 1987. Reprint of the 1934 original.

[14] L. Pandolfi, Stabilization of neutral functional differential equations, J. Optimization Theory Appl., 20 (1976), pp. 191-204.

[15] A. J. Pritchard And J. ZabCZyK, Stability and stabilizability of infinite-dimensional systems, SIAM Rev., 23 (1981), pp. 25-52.

[16] R. RABAH And G. M. SKLyAR, The analysis of exact controllability of neutral-type systems by the moment problem approach, SIAM J. Control Optim., 46 (2007), pp. 2148-2181.

[17] R. Rabah, G. M. Sklyar, and P. Yu. Barkhayev, Stability and stabilizability of mixed retarded-neutral type systems, ESAIM Control Optim. Calc. Var., 18 (2012), pp. 656-692.

[18] R. Rabah, G. M. Sklyar, and A. V. Rezounenko, Generalized Riesz basis property in the analysis of neutral-type systems, C. R. Math. Acad. Sci. Paris, 337 (2003), pp. 19-24.

[19] - Stability analysis of neutral-type systems in Hilbert space, J. Differential Equations, 214 (2005), pp. 391-428.

[20] — On strong regular stabilizability for linear neutral-type systems, J. Differential Equations, 245 (2008), pp. 569-593.

[21] R. Rabah, G. M. Sklyar, And A. V. Rezounenko, On pole assignment and stabilizability of neutral-type systems, in Topics in time delay systems, vol. 388 of Lecture Notes in Control and Inform. Sci., Springer, Berlin, 2009, pp. 85-93.

[22] D. L. Russell, Controllability and stabilizability theory for linear partial differential equations: recent progress and open questions, SIAM Rev., 20 (1978), pp. 639-739.

[23] V. Sinswat AND F. FALlside, Eigenvalue/eigenvector assignment by state-feedback, Internat. J. Control, 26 (1977), pp. 389-403. 
[24] K. V. Sklyar, R. Rabah, And G. M. Sklyar, Eigenvalues and eigenvectors assignment for neutral-type systems, Comptes Rendus Mathématique, 351 (2013), pp. 91-95.

[25] M. Slemrod, A note on complete controllability and stabilizability for linear control systems in Hilbert space, SIAM J. Control, 12 (1974), pp. 500-508.

[26] Byung-Keun Song, S. Jayasuriya, Active vibration control using eigenvector assignment for mode localization. Proc. American Control Conf., pp. 1020-1024, San-Francisco, 1993.

[27] W. M. Wonham, Linear multivariable control: A geometric approach, Springer, New York, 1985.

[28] R. M. Young, An introduction to nonharmonic Fourier series, Academic Press, New York, 1980. 